TI 2013-149/VI

Tinbergen Institute Discussion Paper

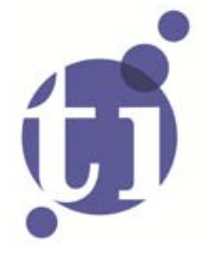

tinbergen
institute

\title{
Participation Constraints in Pension Systems
}

Roel Beetsma',2,3,4,5

Ward Romp'

\footnotetext{
1 Faculty of Economics and Business, University of Amsterdam;

2 Tinbergen Institute;

3 Netspar;

4 CEPR;

${ }^{5}$ CESifo.
} 
Tinbergen Institute is the graduate school and research institute in economics of Erasmus University Rotterdam, the University of Amsterdam and VU University Amsterdam.

More TI discussion papers can be downloaded at http://www.tinbergen.nl

Tinbergen Institute has two locations:

Tinbergen Institute Amsterdam

Gustav Mahlerplein 117

1082 MS Amsterdam

The Netherlands

Tel.: +31(0)205251600

Tinbergen Institute Rotterdam

Burg. Oudlaan 50

3062 PA Rotterdam

The Netherlands

Tel.: +31(0)10 4088900

Fax: $+31(0) 104089031$

Duisenberg school of finance is a collaboration of the Dutch financial sector and universities, with the ambition to support innovative research and offer top quality academic education in core areas of finance.

DSF research papers can be downloaded at: http://www.dsf.nl/

Duisenberg school of finance

Gustav Mahlerplein 117

1082 MS Amsterdam

The Netherlands

Tel.: +31(0)20 5258579 


\title{
Participation Constraints in Pension Systems*
}

\author{
Roel Beetsma ${ }^{\dagger}$ \\ MN Chair in Pension Economics \\ University of Amsterdam \\ CEPR, CESifo, Netspar, Tinbergen Institute \\ Ward Romp $p^{\ddagger}$ \\ University of Amsterdam \\ Tinbergen Institute \\ Netspar
}

This version: September 9, 2013

\begin{abstract}
We explore voluntary participation in pension arrangements. Individuals only participate when participation is more attractive than autarky. The benefit of participation is that risks can be shared with future generations. We apply our analysis to a pay-as-you-go system, a funded system without buffers and a funded system with buffers. Buffers play a particularly interesting role, because they raise the sensitivity of the contributions to the asset returns. In particular, compared to a system without buffer requirements, they require higher contributions when asset returns are low. Moreover, individual contributions may be increasing or decreasing in the size of the young cohort, depending on whether the fund has more or less reserves than required. We confine ourselves to recursive settings and study equilibria characterised by thresholds on the contribution that young generations are prepared to make assuming that the future young apply the same threshold. For standard parameter settings two such equilibria exist, of which only the one with the higher threshold is consistent with the initial young being prepared to start the system. Finally, we explore the social welfare maximising policy parameter settings for various levels of uncertainty and risk aversion.
\end{abstract}

\footnotetext{
${ }^{*}$ We thank for helpful comments and suggestions Jos van Bommel and participants at a Netspar seminar.

${ }^{\dagger}$ Mailing address: MN Chair in Pension Economics, Amsterdam School of Economics, University of Amsterdam, Valckenierstraat 65-67, 1018 XE Amsterdam, The Netherlands; Phone: +31.20.5255280; fax: +31.20.5254254; e-mail: R.M.W.J.Beetsma@uva.nl.

${ }^{\ddagger}$ Mailing address: Amsterdam School of Economics, University of Amsterdam, Valckenierstraat 65-67, 1018 XE Amsterdam, The Netherlands; Phone: +31.20.5257344; fax: +31.20.5254254; e-mail: W.E.Romp@uva.nl.
} 


\section{Introduction}

Around the world population ageing is putting collective pension arrangements under financial pressure. While participation is often mandatory, working-age participants are becoming increasingly reluctant to contribute to such arrangements out of fear that, once they have retired themselves, insufficient resources may have been left for their own pension, while future workers will refuse to make up for the shortages. These fears are particularly strong among the youngest workers. Of course, once large groups of participants prefer to leave the system they participate in, its demise becomes imminent. Hence, if this danger looms, it is important to start rethinking the system's design, such that those participants are prepared to stay on board.

This paper explores the sustainability of pension arrangements when participation is voluntary; for those arrangements that are sustainable it characterises the equilibria that arise. The benefit of participation is that risks can be shared among the different generations in the system. Real-world experience suggests that the benefits from intergenerational risk sharing are nontrivial. For example, assuming that the contributions are invested in the financial markets, Burtless (2000) shows that the annuity benefit of a male worker who entered an individual retirement account plan in 1975 at the age of twenty-two and retires at the age of 62 can expect to receive only two-fifths of what he could have expected to receive when entering in 1969 and retiring at the same age.

We set up an overlapping-generations model in which individuals live for two periods. We allow for two sources of uncertainty, namely demographic risk, as captured by the birth rate of young individuals, and financial market risks, captured by the return on savings. In the first period of their life individuals decide whether to participate in the pension arrangement. They do so when the expected utility under participation exceeds that under autarky, i.e. when they do not participate. By participating risks during retirement can be shared with the future young. Limiting ourselves to recursive settings in which each generation faces the same decision problem, this decision translates into a threshold on the contribution that the young need to make, given their belief about the threshold of the future young. We confine ourselves to the study of equilibria in which, given the equilibrium belief about the future threshold, the system persists if the contribution is lower than the threshold, while it collapses when the contribution exceeds the threshold. In addition, we are only interested in equilibria that are stable.

Our set-up can can be applied to a wide range of pension arrangements. We consider three specific, but important, arrangements. The first is a defined-benefit pay-as-you-go (PAYG) scheme. The scheme has no assets, because each period aggregate benefits equal aggregate contributions. We show that for plausible parameter settings, there is a wide range of contribution payments consistent with the continuation of the system. Hence, a near-future collapse is unlikely. The second application considers a pension fund without any buffers, while the third application generalises this arrangement to one with buffers. Imposing a buffer requirement has interesting consequences for the sustainability of a funded pension arrangement. If the asset returns are high, the buffer creates some 'free' money that can be used to lower the contributions by the young. However, if the asset returns are low, the incoming cohort not only has to guarantee the pension benefits of the retired, but it also has to replenish the buffer, implying a contribution that is actually higher than in the case without buffers. Overall, the sensitivity of the contributions to the asset returns increases 
when buffer requirements are higher. Moreover, individual contributions may be increasing or decreasing in the size of the young cohort, depending on whether the fund has more or less reserves than required. When the fund has more reserves than required, the excess reserves per young individual are higher when the young generation is smaller and, hence, the contribution can be reduced. However, when reserves are too low, a smaller young generation requires a larger increase in the individual contribution to replenish the reserves.

For standard parameter values we find that each of the three pension arrangements features two equilibria, one with a lower and one with a higher threshold. We also explore the policy parameter settings that maximise social welfare. Here, we confine ourselves to equilibria that are consistent with the initial young benefiting from starting the system. This implies that for each arrangement we can limit ourselves to the one with the higher threshold. An increase in demographic uncertainty has only minor effects on the system's design parameters, because demography does not directly affect individual utility. Both an increase in financial market uncertainty and an increase in risk aversion raise the benefit from risk sharing, hence the optimal pension benefit and, along with it, the maximum willingness of the young to contribute rise. For funded systems, higher risk aversion lowers the optimal minimum return on the pension contributions. The cost of a fund collapse becomes higher, hence the chance that the young are not willing to guarantee this minimum return should be reduced. Owing to the rather large difference between the expected financial return and the expected population growth rate, the welfare gains from participating in the optimal funded scheme are substantially larger than from participating in the optimal PAYG scheme. Imposing a buffer requirement on the funded scheme raises social welfare further, though only by a relatively small amount, because the participation benefit of the first young generation falls.

The literature exploring voluntary participation in pension arrangements is still very limited. Closest to this paper is Beetsma et al. (2012), who study voluntary participation in a funded pension arrangement without pension buffers and with only uncertainty in the asset returns. They show that the scope for voluntary participation is rather limited, because it can only occur when financial market uncertainty is sufficiently strong and the risk aversion of the participants is sufficiently high. However, whenever an equilibrium with voluntary participation does exist, it never breaks down. The set up studied in this paper is much more general and the analysis is richer. We explore voluntary participation in a wider range of pension systems and pay particular attention to the role of buffers in funded arrangements. We also allow for demographic uncertainty. Moreover, the equilibria are characterised in a different way, while we study equilibria in which the probability of a collapse of the pension system is non-zero each period.

Only a few other papers investigate discontinuity risk in funded pension systems. Demange and Laroque (2001) analyse risk sharing in a PAYG system with voluntary contributions. Bovenberg et al. (2007) explore the problem of negative buffers that may deter new cohorts from entering the pension arrangement, while Demange (2009) investigates political sustainability risk in a PAYG system. Westerhout (2009) quantifies the feasible amount of risk-sharing when the old are bound by their pension contract, while the young are free to choose whether they will participate. The latter refuse to join the pension fund when it is under financial distress. Unlike in this paper, Westerhout (2009) assumes that the return on the funds' assets exceeds that on private savings, which 
makes it relatively attractive to join the fund. Finally, Molenaar et al. (2011) explore the threshold ratio of assets over liabilities for which it is optimal for a participant to quit a pension fund. Their model differs substantially from ours and is designed to capture the main features of a Dutch DB pension fund. Moreover, in contrast to this paper, there is no fully-fledged analysis of the existence and characterisation of equilibria with voluntary participation.

There exists a rather large literature exploring risk sharing within mandatory pension arrangements. Hence, this literature does not address the decision to participate in these schemes. Intergenerational risk sharing in pay-as-you-go (PAYG) pension schemes is analysed by Enders and Lapan (1982), Thogersen (1998), Bohn (1999, 2003), Wagener (2004), Sanchez-Marcos and SanchezMartin (2006), Gottardi and Kubler (2006), Krueger and Kubler (2006), Ball and Mankiw (2007) and Olovsson (2010). Gollier (2008), Cui et al. (2011), Mehlkopf (2012) and Bonenkamp and Westerhout (2013) explore intergenerational risk sharing in funded pension arrangements. Hassler and Lindbeck (1997), De Menil and Sheshinsky (2003) and Matsen and Thogersen (2004) analyse the trade off between PAYG and pension funding, while Beetsma and Bovenberg (2009) and Beetsma et al. (2013) investigate intergenerational risk sharing when a PAYG and a funded pension are combined.

The remainder of this paper is structured as follows. Section 2 presents the model. Section 3 characterises the participation decision. In Section 4 we explore three common applications: a PAYG arrangement, a pension fund without buffers and a pension fund with buffers. Finally, Section 6 concludes the main body of the paper. The Appendix contains some proofs.

\section{The Model}

We present a simple overlapping generations model in which individuals live for two periods. They receive an exogenous endowment income in the first period of their life and decide whether or not to participate in a pension arrangement. In addition, they decide about their level of "personal savings", i.e. the amount of savings outside the pension arrangement in which they potentially participate. In the second period of their life they consume the proceeds of their savings and their pension benefit in case they decided to participate.

\subsection{Individuals}

Throughout this paper cohort-specific variables have two subscripts. The first subscript indicates the year of birth, while the second subscript denotes the current period. We refer to the cohort born in period $v$ as "cohort $v$ ". At birth, individuals optimise expected lifetime utility:

$$
\mathcal{U}_{v}=u\left(c_{v, v}\right)+\beta \mathrm{E}_{v}\left[u\left(c_{v, v+1}\right)\right]
$$

where $\beta$ denotes the discount factor. The utility function fulfils the usual properties $u^{\prime}(\cdot)>0$, $u^{\prime \prime}(\cdot)<0$ and $\lim _{c \downarrow} u^{\prime}(c)=\infty$. At birth the individual receives an exogenous endowment $y$, which for simplicity we assume to be the same irrespective of the period of birth.

In the first period of life an individual decides whether or not to participate in a pension scheme. He can make this decision only once. That is, if he opts in, he cannot opt out later, while if he opts 
out, he has to stay out for his entire life and spend his life in autarky. In particular, by choosing to participate the individual will be obliged to participate not only in the first period of his life, but also in the second period of his life. We can think of participation as signing a legally-binding contract that prohibits leaving the system when retired. Specifically, this implies that it is not possible for the elderly participants to dismantle the system and distribute its assets, if any, among themselves. In the first period of his life, an individual also decides about his current level of consumption, hence about his personal savings. In the following we will denote variables under autarky by superscript $a$, while variables under participation carry no superscript.

\subsubsection{Autarky}

Consider an individual born in period $t$. If he decides not to participate in the pension arrangement he divides his initial endowment $y$ between consumption and savings. The latter generate a stochastic rate of return $r_{t+1}$, so consumption when old in autarky is

$$
c_{t, t+1}^{a}=\left(1+r_{t+1}\right) s_{t}^{a}
$$

where $s_{t}^{a}=y-c_{t, t}^{a}$ is savings under autarky .

Optimisation given the budget constraint (2) yields the usual Euler equation

$$
u^{\prime}\left(c_{t, t}^{a}\right)=\beta \mathrm{E}_{t}\left[\left(1+r_{t+1}\right) u^{\prime}\left(c_{t, t+1}^{a}\right)\right]
$$

which, together with the budget constraint, determines the consumption profile. We denote expected utility for an individual born in period $t$ in autarky by $\mathcal{U}_{t}^{a}$.

\subsubsection{Participation}

If the individual born in period $t$ decides to participate in the already existing pension arrangement, he has to pay a contribution $\tau_{t}$ to the pension arrangement. This contribution is determined by the state of the economy and the arrangement according to a system-specific rule. In Section 4 we will provide three examples of such rules. The individual's savings are given by

$$
s_{t}=y-c_{t, t}-\tau_{t}
$$

The benefit of participation is that the pension system may provide some insurance against particular bad shocks during the retirement period. However, the payout of the pension system depends on whether the next generation is also willing to participate. The simplest example is a PAYG system. If the next generation opts out, the pension benefit for the current generation is zero. If the next generation decides to participate, the current young receive a pension benefit $\theta_{t+1}$ when they are old. This benefit depends on the particular type of pension arrangement and it may depend on the future state of the economy. Hence, assuming that the next generation participates, consumption of cohort $t$ when it is old is

$$
c_{t, t+1}=\left(1+r_{t+1}\right) s_{t}+\theta_{t+1} .
$$


The pension benefit $\theta_{t+1}$ is financed out of the next cohort's contribution $\tau_{t+1}$ and the possible excess reserves in the pension system.

If the next cohort born in period $t+1$ decides not to participate, then, if there are any pension reserves, these are evenly distributed over the existing participants. Because in our simple set-up there is only one remaining cohort of participants, namely those born in $t$, there is no conflict over the assignment of property rights to the pension reserves. In this case, cohort $t$ 's consumption in period $t+1$ is

$$
c_{t, t+1}^{c}=\left(1+r_{t+1}\right) s_{t}+\theta_{t+1}^{c},
$$

where a superscript $c$ indicates a collapse of the system. Hence, $c_{t, t+1}^{c}$ is consumption of an old individual born in period $t$ after the system collapses. After a collapse, the pension system is liquidated and the revenues are used to $\operatorname{cover} \theta^{c}$. For many systems, such as funded pension systems, $\theta_{t+1}^{c}$ is strictly positive and it may even exceed $\theta_{t+1}$. This implies that under special circumstances the old generation may even prefer a collapse of the system. However, the obligation not leave the system prevents the old generation from liquidating it when the young decide to participate.

The value of participation, $\mathcal{U}^{p}$, depends on the current state of the economy, which determines the current contribution, and the expected pension benefit when old. This last benefit depends on the willingness of future generations to participate. We assume that there exists a threshold contribution level above which the next generation decides not to participate. We denote the beliefs of cohort $t$ about the threshold of cohort $t+1$ by $\tau_{t}^{*}$. Cohort $t$ 's expected utility of participation is

$$
\mathcal{U}_{t}^{p}=u\left(c_{t, t}\right)+\beta \int_{D^{c}\left(\tau_{t}^{*}\right)} u\left(c_{t, t+1}^{c}\right) p(\phi)+\beta \int_{D\left(\tau_{t}^{*}\right)} u\left(c_{t, t+1}\right) p(\phi),
$$

with $D\left(\tau^{*}\right)=\left\{\phi \mid \tau(\phi) \leq \tau^{*}\right\}$ all states in which the system continues and $D^{c}\left(\tau^{*}\right)=\left\{\phi \mid \tau(\phi)>\tau^{*}\right\}$ its complement, i.e. all states in which the system collapses. Since the pension system does not distort the inter-temporal consumption-savings trade-off, the first-order condition determining consumption in the first period of life is

$$
u^{\prime}\left(c_{t, t}\right)=\beta \int_{D^{c}\left(\tau_{t}^{*}\right)}\left(1+r_{t+1}\right) u^{\prime}\left(c_{t, t+1}^{c}\right) p(\phi)+\beta \int_{D\left(\tau_{t}^{*}\right)}\left(1+r_{t+1}\right) u^{\prime}\left(c_{t, t+1}\right) p(\phi) .
$$

This is the standard Euler equation, taking the possibility of a collapse into account.

\subsection{The demography}

We denote the size of the current old generation by $N_{t-1}$. This generation gives birth to a new generation of size $N_{t}$ at the start of the current period. The gross birth rate $b_{t} \equiv N_{t} / N_{t-1}$ is stochastic. It is also the inverse of the old-age dependency ratio $\gamma_{t} \equiv N_{t-1} / N_{t}$. Population growth is $n_{t}=N_{t} / N_{t-1}-1$. Hence, $1+n_{t}=b_{t}$.

\subsection{The pension system}

The pension system collects contributions from the current young and pays pension benefits to the current old. Depending on the system, it may or may not manage assets and run a surplus or a 
deficit. If the new generation participates, total assets $(A)$ of the pension fund evolve as

$$
A_{t+1}=\left(1+r_{t+1}\right)\left[A_{t}+N_{t} \tau_{t}-N_{t-1} \theta_{t}\right]
$$

It is often easier to focus on assets per current young person who participates. Dividing both sides by $N_{t}$ and using the definition of the old-age dependency ratio gives

$$
a_{t+1}=\gamma_{t+1}\left(1+r_{t+1}\right)\left(a_{t}+\tau_{t}-\gamma_{t} \theta_{t}\right)
$$

where $a_{t} \equiv A_{t} / N_{t}$ and $\tau_{t}$ and $\theta_{t}$ depend on the specific pension arrangement and the state of the economy.

If the system collapses, all the assets still in the fund are liquidated and divided over the current old generation. Hence,

$$
\theta_{t}^{c}=a_{t} / \gamma_{t}
$$

\subsection{The economy}

The relevant variables for the participation decision of the newborns are the asset return, the birth rate and the asset position of the pension arrangement. At the time of the participation decision, the young generation knows the current old-age dependency ratio and the current assets of the system. Equation (10) shows that in general the asset position of the system features an auto-regressive component. Below we will see that we can only solve our model if the pension arrangement is such that this auto-regressive component disappears. The examples that we study later fulfil this requirement.

\section{The Participation Decision}

A newborn in period $t$ decides to participate if $\mathcal{U}_{t}^{p}>\mathcal{U}_{t}^{a}$ given the current state of the economy (including the pension system) $\phi_{t}$ and his belief about the threshold for participation $\tau_{t}^{*}$ of the next young generation. This dependence on what the current generation believes about the next generation's threshold makes it impossible to solve this model in its most general set up. Therefore, we focus on situations in which the problem of solving the model is recursive. In other words, we limit ourselves to cases in which each generation faces the same decision problem. To this end, we assume that the next period's asset return and birth rate do not depend on the current state of the economy. ${ }^{1}$ In this setting, expected utility under autarky is constant, because the probability density function of the return on savings is time invariant. Furthermore, we focus on pension arrangements for which the asset position always returns to the same specific value. That is, we restrict the pension system to one in which $a_{t}+\tau_{t}-\gamma_{t} \theta_{t}$ is constant. Hence, once in a given period the cash-flows associated with the system have taken place, the system's assets must be constant.

For the sake of readability we can now drop time subscripts and use an apostrophe (e.g. $\phi^{\prime}$ ) to denote the next period's values. We write consumption when young as $c_{1}$ and consumption when old as $c_{2}$. The next period's state $\phi^{\prime}$ is independent of the current state $\phi$. Hence, all relevant

\footnotetext{
${ }^{1}$ Because a period in our model corresponds to the length of a generation, this assumption does not seem to be too unrealistic.
} 
information for the individual about the current state is captured by the required contribution $\tau(\phi)$. In turn, this implies that the expected utility of participation $\mathcal{U}^{p}$ is fully determined by $\tau$ and the belief $\tau^{*}$. To make this dependence explicit we write

$$
\begin{aligned}
\mathcal{U}^{p}\left(\tau, \tau^{*}\right)=\max _{c_{1}, s} u( & \left.c_{1}\right)+\beta \int_{D\left(\tau^{*}\right)} u\left[\left(1+r\left(\phi^{\prime}\right)\right) s+\theta\left(\phi^{\prime}\right)\right] p\left(\phi^{\prime}\right) \\
& +\beta \int_{D^{c}\left(\tau^{*}\right)} u\left[\left(1+r\left(\phi^{\prime}\right)\right) s+\theta^{c}\left(\phi^{\prime}\right)\right] p\left(\phi^{\prime}\right), \\
\text { s.t. } s= & y-c_{1}-\tau,
\end{aligned}
$$

where we have substituted $c_{2}$ out using the second period's budget constraints (5) and (6).

This function has the following properties (see the Appendix for the derivation)

$$
\begin{aligned}
\frac{\partial \mathcal{U}^{p}}{\partial \tau} & =-u^{\prime}\left(c_{1}\right)<0, \\
\frac{\partial \mathcal{U}^{p}}{\partial \tau^{*}} & =\beta \int_{\partial D\left(\tau^{*}\right)}\left[u\left(c_{2}^{c}\right)-u\left(c_{2}\right)\right] p\left(\phi^{\prime}\right) \cdot z \lessgtr 0,
\end{aligned}
$$

where $\partial D\left(\tau^{*}\right)$ denotes the boundary of $D\left(\tau^{*}\right)$ and $z$ the velocity vector at the boundary.

A higher contribution $\tau$ lowers lifetime income and, hence, as equation (13) shows, always lowers lifetime utility. The effect of a higher threshold is ambiguous in general. A higher $\tau^{*}$ always increases the region over which the system survives, but at the boundary consumption after a collapse of the system may or may not exceed consumption under participation. In section 4.3 we present a pension system in which the pension fund has a buffer. As long as the fund has a positive buffer, the old actually prefer the young not to participate, so that fund can be closed and they can claim these reserves for themselves. Hence, in general, the utility effect of a higher contribution threshold is ambiguous.

The key to determining the current generation's optimal choice - to participate or not - is their belief about the contribution threshold of the next generation, $\tau^{*}$. Given our recursive set-up, each generation faces the same decision. Therefore, we assume that a specific belief $\hat{\tau}$ about the future threshold is a valid threshold if it is also a valid threshold for the current generation. We define an equilibrium as:

Definition. An equilibrium is a savings rule for consumers under participation $s(\tau)$, a savings rule in autarky $s^{a}$, and a threshold $\hat{\tau}$, such that for these savings rules and threshold (1) the Euler equations in autarky and under participation hold, (2) the budget constraints in autarky and under participation hold, and for this threshold the following conditions hold:

1. for all $\tau>\hat{\tau}, \mathcal{U}^{p}(\tau, \hat{\tau})<\mathcal{U}^{a}$,

2. for all $\tau<\hat{\tau}, \mathcal{U}^{p}(\tau, \hat{\tau})>\mathcal{U}^{a}$,

3. it must be 'stable', i.e. $\left|\mathcal{U}_{2}^{p}\right|<\left|\mathcal{U}_{1}^{p}\right|$, in $\tau=\tau^{*}=\hat{\tau}$,

where $\mathcal{U}_{1}^{p}$ and $\mathcal{U}_{2}^{p}$ are the first derivatives of $\mathcal{U}^{p}\left(\tau, \tau^{*}\right)$ with respect to its first and second argument, respectively. 
For interior values of $\tau$, the first two requirements simply boil down to $\mathcal{U}^{p}(\hat{\tau}, \hat{\tau})=\mathcal{U}^{a}$. To accommodate corner solutions, we have to split this condition into two parts. Condition 1 states that it is optimal for the young who believe that $\hat{\tau}$ is a threshold for the next generation, to not participate in the system (since $\mathcal{U}^{p}<U^{a}$ ) whenever the required contribution exceeds this threshold. Below we will consider pension arrangements that require a minimum contribution level and where this minimum turns out to be an equilibrium threshold. A minimum value $\tau_{\min }$ of $\tau$ fulfils Condition 1 if $\mathcal{U}^{p}\left(\tau_{\text {min }}, \tau_{\text {min }}\right) \leq \mathcal{U}^{a}$, since $\mathcal{U}^{p}$ is continuous and decreasing in the first argument. Thus, this condition could be relevant if there is a non-zero probability that the contribution is exactly equal to this minimum value. The fact that we observe a pension system then merely indicates that the contribution has never been higher than this minimum level. Condition 2 says that it is optimal for the young who believe that $\hat{\tau}$ is a threshold for the next generation to participate (since $\mathcal{U}^{p}>U^{a}$ ) whenever the required contribution falls below this threshold.

Condition 3 requires more explanation. The purpose of this condition is to exclude 'silly' or 'unstable' equilibria as identified by Beetsma et al. (2012). Stability means that a young who happens to have a belief about the next generation's threshold $\tau^{0}$ close to $\hat{\tau}$ and tries to determine the threshold contribution that makes him indifferent between participation and autarky given his belief $\tau^{0}$, eventually ends up in the equilibrium $\hat{\tau}$ by using this threshold as a belief. Formally, the threshold $\tilde{\tau}$ of a young who has a belief $\tau^{*}$ is defined by the implicit equation $\mathcal{U}^{p}\left(\tilde{\tau}, \tau^{*}\right)=\mathcal{U}^{a}$. Hence, we can write $\tilde{\tau}=\tilde{\tau}\left(\tau^{*}\right)$. Starting at $\tau^{*}=\tau^{0}$ in the neighbourhood of $\hat{\tau}$ yields the sequence $\tau^{1}=\tilde{\tau}\left(\tau^{0}\right), \tau^{2}=\tilde{\tau}\left(\tau^{1}\right)$, etc. This series converges to $\hat{\tau}$ if $\left|\partial \tilde{\tau} / \partial \tau^{*}\right|<1$ in $\tilde{\tau}=\hat{\tau}$. Implicit differentiation yields $\partial \tilde{\tau} / \partial \tau^{*}=-\mathcal{U}_{2}^{p} / \mathcal{U}_{1}^{p}$. Hence, a fixed point $\hat{\tau}$ is stable if $\left|\mathcal{U}_{2}^{p}\right|<\left|\mathcal{U}_{1}^{p}\right|$ in this point, which is the third condition.

To calculate equilibrium thresholds we define

$$
\Delta^{p}(\tau) \equiv \mathcal{U}^{p}(\tau, \tau)-\mathcal{U}^{a}
$$

Finding a threshold $\hat{\tau}$ boils down to a simple one-dimensional root finding problem, as well as checking the values of $\Delta^{p}$ at potential corner solutions. The derivative of $\Delta^{p}$ is

$$
\frac{d \Delta^{p}}{d \tau}=\mathcal{U}_{1}^{p}(\tau, \tau)+\mathcal{U}_{2}^{p}(\tau, \tau)
$$

Given that $\mathcal{U}_{1}^{p}<0$ (see (13)), Condition 3 implies that any root $\hat{\tau}$ of $\Delta^{p}$ on an upward sloping part of the function cannot be a stable equilibrium and can be discarded. Thus, a negative first derivative of $\Delta^{p}(\cdot)$ provides only a necessary, but not a sufficient, condition for the stability of the root.

Our equilibrium concept quite closely resembles that of the "organizational equilibrium" proposed by Prescott and Rios-Rull (2005). In the context of our model, their so-called "no-restarting condition" says that the current threshold can only be an equilibrium threshold if, for a state of the economy identical to the current one, the next young generation cannot attain a higher utility than the current young generation by abolishing the system and restarting it with a different threshold. Our concept differs from theirs in that, once the threshold has been exceeded, the arrangement disappears forever. 


\section{Three applications}

In this section we will use the above model to analyse three specific pension arrangements, focussing on the possibility that a new young generation decides not to participate. The above analysis can be applied, because in all three applications the assets managed within the system return to the same constant value.

In all our applications we will assume the same structure of the underlying economy with the same underlying uncertainties. Given our two-cohort model, one period consists of 30 years. The initial endowment $y$ is normalised to 1 . Following Beetsma et al. (2012) we model the gross return $(1+r)$ as a $\log$-normal process $\operatorname{LogN}\left(\mu_{r}, \sigma_{r}^{2}\right)$. We make use of Campbell et al. (2003) for the calibration of the portfolio return. The risk free interest rate is set to $2.1 \%$ and the risk premium on equity is $6.8 \%$, with a standard deviation of $18.2 \%$. Given the long investment horizon of individuals we set the share invested in equity at $75 \%$. Inflation is set at $2.0 \%$. Hence, the mean annual real return on the whole portfolio is $5.2 \%$. For a 30 year period and a log-normal distribution for the gross real return, this translates into $(1+r) \sim \operatorname{LogN}(1.27,0.71)$.

The birth rate also follows a log-normal distribution, calibrated to the average annual population growth and standard deviation in the Netherlands from 1900 to 2012. The average annual population growth was $1.06 \%$, with a standard deviation of $0.47 \%$. For a 30 -year period, we have $b \sim \operatorname{LogN}(0.32,0.026)$. For simplicity, we assume that both processes are independent of each other, so the joint probability density function $p(r, b)$ can be written as $p(r, b)=p_{r}(r) p_{b}(b){ }^{2}{ }^{2}$ Further, for utility we assume a standard CRRA felicity function

$$
u(c)=\frac{c^{1-\rho}}{1-\rho},
$$

where we set $\rho=5$ in our baseline simulations. Finally, we set the discount factor over our 30-year period at 0.5 , which translates into an annual discount factor of roughly 0.977 .

\subsection{Defined benefit pay-as-you-go}

In a PAYG system, the pension benefits of the old generation are fully covered by the contributions of the young. Hence, $a_{t}=0$. For simplicity, we assume that the system is of a defined-benefit type with a fixed pension benefit $\theta$. The contribution paid by new entrants varies to absorb all shocks:

$$
\tau_{t}=\gamma_{t} \theta=\theta / b_{t}
$$

\footnotetext{
${ }^{2}$ Systematic empirical evidence on the relationship between population growth and the equity premium seems rather hard to obtain. Yu (2002) finds evidence of a positive relationship between population growth and bond and stock returns for both small and large companies in the U.S., but unfortunately does not present direct evidence of the relationship between the equity premium and population growth. More work has been done on the study of the relationship between the age composition of the population and the equity premium. Geanakoplos et al. (2004) report a higher equity premium for the U.S. when the ratio of the middle-aged to the young is relatively low, while evidence for other large developed countries suggests that an increasing ratio of middle-aged to young is accompanied by high stock market returns over the period of the nineteen eighties and nineties. Kuhle et al. (2007) present a quantitative theoretical analysis for the U.S. that suggests an increase in the equity premium when a relatively small young cohort enters the labour market. In view of the lack of a consensus figure for the correlation between population growth and the equity premium, we stick to our assumption that the two are uncorrelated.
} 
Since $b \sim \operatorname{LogN}\left(\mu_{b}, \sigma_{b}^{2}\right)$, the contribution $\tau$ follows $\operatorname{LogN}\left(-\mu_{b}+\log (\theta), \sigma_{b}^{2}\right)$ and is in principle only bounded from below. The lifetime budget constraint imposes an upper limit on the contribution. If the young decide not to participate, the old get nothing, because there are no pension assets. Hence, any contribution higher than $y$ violates the lifetime budget constraint when the system collapses in the next period, so may be excluded from the analysis. Savings must be positive to ensure positive consumption when old if the system collapses. Moreover, they cannot exceed $y-\tau_{t}$ to prevent negative consumption when young.

Given this specification, we can $\operatorname{write} \mathcal{U}^{p}(\cdot, \cdot)$ as

$$
\begin{aligned}
\mathcal{U}^{p}\left(\tau, \tau^{*}\right)=\max _{s} u(y-s-\tau)+\beta \int_{D\left(\tau^{*}\right)} u\left[\left(1+r^{\prime}\right) s+\theta\right] p\left(\phi^{\prime}\right) & \\
& +\beta \int_{D^{c}\left(\tau^{*}\right)} u\left[\left(1+r^{\prime}\right) s\right] p\left(\phi^{\prime}\right) .
\end{aligned}
$$

The contribution depends only on the birth rate, so given a threshold $\tau^{*}$, there is a threshold for the birth rate $b^{*}=\theta / \tau^{*}$ below which the system collapses, that is, $D=\left\{r^{\prime}, b^{\prime} \mid b^{\prime}>\theta / \tau^{*}\right\}$ and $D^{c}=\left\{r^{\prime}, b^{\prime} \mid b^{\prime} \leq \theta / \tau^{*}\right\}$.

For this pension system, $\Delta^{p}$ can be written as

$$
\begin{aligned}
\Delta^{p}(\tau)=\max _{s} u(y-s-\tau)+\beta \int_{-1}^{\infty} \int_{\theta / \tau}^{\infty} u\left[\left(1+r^{\prime}\right) s+\theta\right] p_{r}\left(r^{\prime}\right) p_{b}\left(b^{\prime}\right) d b^{\prime} d r^{\prime} \\
\\
\quad+\beta \int_{-1}^{\infty} \int_{0}^{\theta / \tau} u\left[\left(1+r^{\prime}\right) s\right] p_{r}\left(r^{\prime}\right) p_{b}\left(b^{\prime}\right) d b^{\prime} d r^{\prime}-\mathcal{U}^{a}
\end{aligned}
$$

Changing the order of integration gives

$$
\Delta^{p}(\tau)=\max _{s} u(y-s-\tau)+\beta\left(\Omega_{0}(s)-P_{b}(\theta / \tau) \Omega_{1}(s)\right)-\mathcal{U}^{a},
$$

where $P_{b}(\cdot)$ is the cumulative density function of $b, \Omega_{0}(s) \equiv \mathrm{E}_{r}\left(u\left[\left(1+r^{\prime}\right) s+\theta\right]\right), \Omega_{1}(s) \equiv \mathrm{E}_{r}(u[(1+$ $\left.\left.\left.r^{\prime}\right) s+\theta\right]-u\left[\left(1+r^{\prime}\right) s\right]\right)>0$, and $\mathrm{E}_{r}(\cdot)$ is the expected value with respect to $r^{\prime}$.

In this PAYG system we have $\Delta^{p}(0)=0, \lim _{\tau \rightarrow y} \Delta^{p}(\tau)<0, \frac{d \Delta^{p}(\tau)}{d \tau}=-\infty$ as $\tau \rightarrow y$ and $\Delta^{p}$ is strictly downward sloping at $\tau=0$ (see the Appendix for details). Continuity implies that $\Delta^{p}(\tau)=0$ has, besides the solution $\tau=0$, zero roots or an even number of roots, which always come in pairs of an unstable and a stable solution. Numerical results show that for any reasonable parameter constellation $\Delta^{p}(\cdot)$ has at most one such pair. At low values of $\tau$, the wealth effect of an increase in $\tau$ dominates its insurance effect and utility is decreasing in $\tau$. Raising $\tau$ leads to a decreasing probability of a collapse and this effect may at some point start to dominate the wealth effect, leading to positive values of $\Delta^{p}(\cdot)$. However, for large enough $\tau$, the wealth effect always dominates and $\Delta^{p}(\cdot)$ is negative. This effect is enforced by $\Omega_{1}(s)$, since this term grows in size as savings become smaller due to a larger contribution.

Figure 1 shows $\Delta^{p}$ for values of $\tau$ ranging from 0 to 0.3 , i.e. $30 \%$ of the first period's endowment $y$, and a pension benefit $\theta$ of 0.1 , i.e. $10 \%$ of the initial endowment. For this parameter constellation, the function $\Delta^{p}(\tau)$ has three roots: the trivial one at $\tau=0$, an unstable root at 0.07 and a stable root at 0.17 . Given our parameter values, there is a nearly-zero probability that the required 

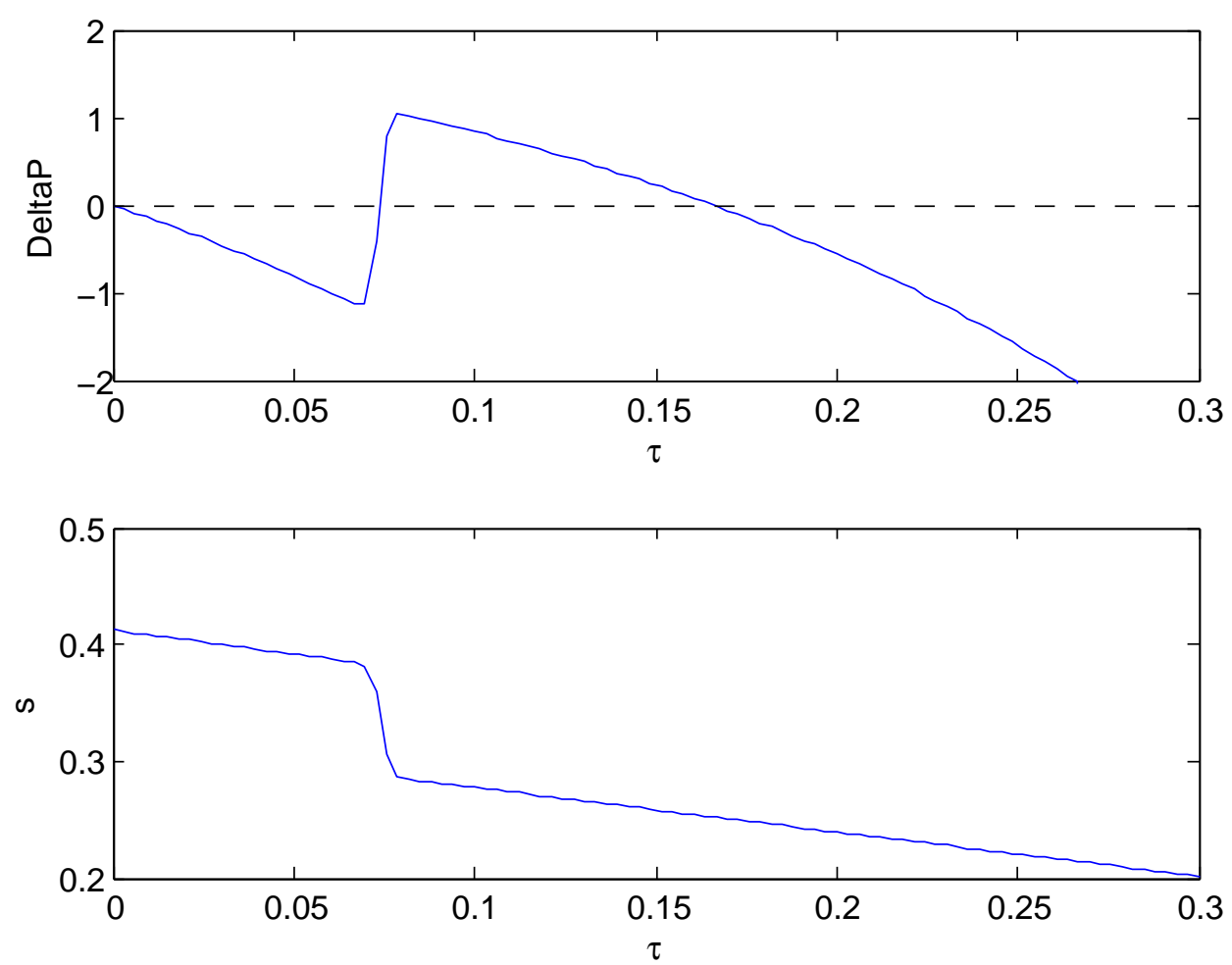

Figure 1: $\Delta(\tau)$ and savings for the PAYG system

contribution is higher than the threshold of 0.17 . Hence, there are two possibilities: either the system collapses immediately or a collapse of this PAYG system within a reasonable time horizon is highly unlikely.

\subsection{Minimum return on pension contribution}

Beetsma et al. (2012) study a system in which a pension fund guarantees a minimum return $r^{*}$ on a basic pension contribution $\zeta$. The pension benefit of the old generation when the system persists is

$$
\theta_{t}=\left\{\begin{array}{ll}
\left(1+r_{t}\right) \zeta & \text { if } r_{t}>r^{*} \\
\left(1+r^{*}\right) \zeta & \text { if } r_{t} \leq r^{*}
\end{array} .\right.
$$

The pension fund keeps no buffers. Hence, if the return on its investments exceeds $r^{*}$, the fund has just enough to pay the pension benefits. If the return on investment is lower than the minimum return $r^{*}$, the young must make an additional contribution that depends on the deficit of the fund. Therefore, the total contribution by the young is

$$
\tau_{t}= \begin{cases}\zeta & \text { if } r_{t}>r^{*} \\ \zeta+\gamma_{t}\left(r^{*}-r_{t}\right) \zeta & \text { if } r_{t} \leq r^{*}\end{cases}
$$

Hence, the total contribution is at least the basic contribution $\zeta$. Like the PAYG system above, this system is also fully recursive. If the young participate, they replenish the assets managed by the 

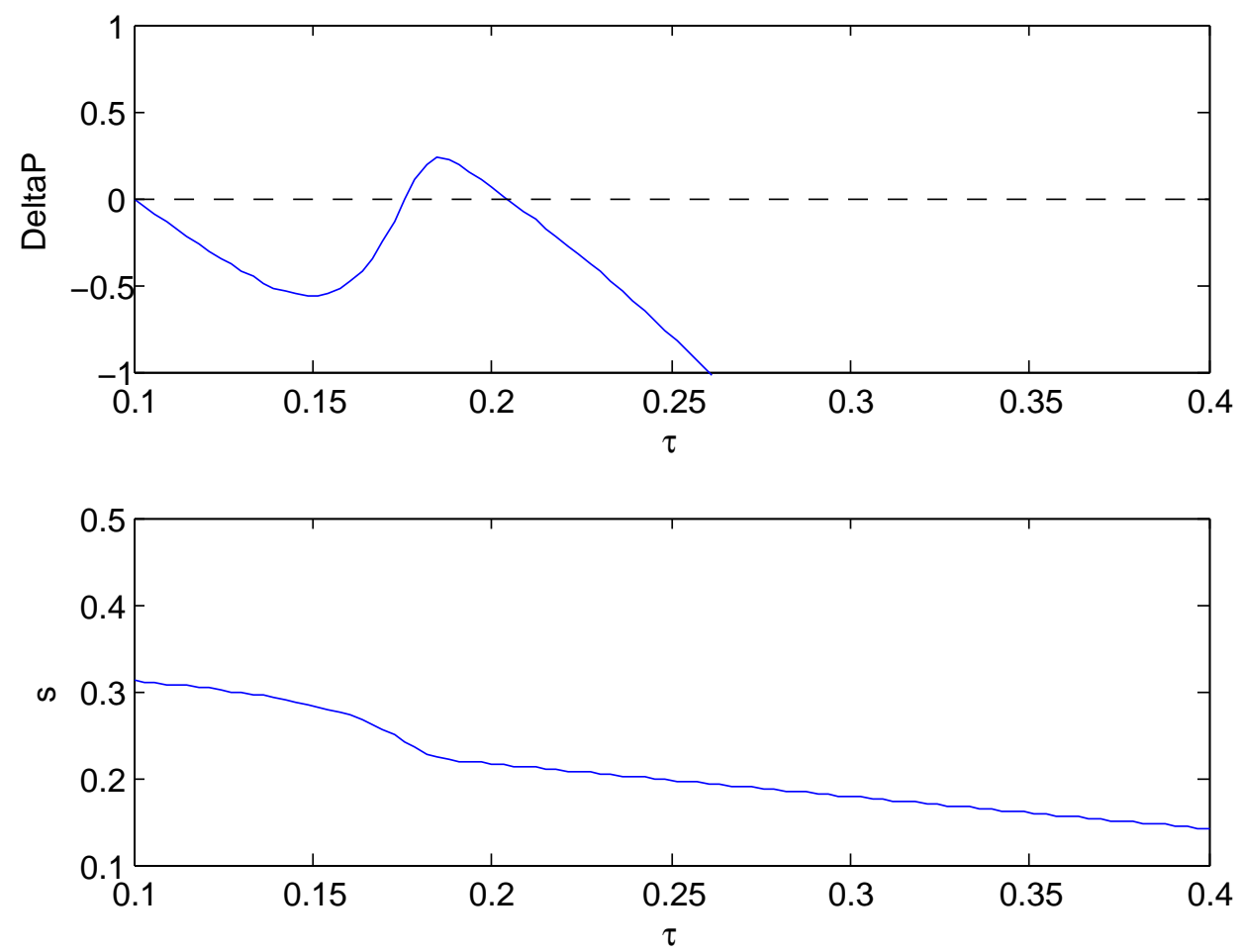

Figure 2: $\Delta(\tau)$ and savings for the funded system without buffers

pension fund to $\zeta$ since $a_{t}+\tau_{t}-\gamma_{t} \theta_{t}=\zeta^{3}{ }^{3}$ The pension benefit of an old individual if the new young decide not to participate is

$$
\theta_{t}^{c}=\left(1+r_{t}\right) \zeta
$$

Given that the contribution is at least equal to $\zeta$ according to (23), we can ignore cases in which $\tau^{*}<\zeta$. This allows us to write

$$
\begin{aligned}
\mathcal{U}^{p}\left(\tau, \tau^{*}\right)=\max _{s} u(y-s-\tau)+\beta \int_{D_{1}\left(\tau^{*}\right)} u[(1 & \left.\left.+r^{\prime}\right) s+\left(1+r^{*}\right) \zeta\right] p\left(\phi^{\prime}\right) \\
& +\beta \int_{D_{2}\left(\tau^{*}\right) \cup D^{c}\left(\tau^{*}\right)} u\left[\left(1+r^{\prime}\right)(s+\zeta)\right] p\left(\phi^{\prime}\right),
\end{aligned}
$$

where $D_{1}\left(\tau^{*}\right)=\left\{r^{\prime}, b^{\prime} \mid r^{\prime}<r^{*},\left(r^{*}-r^{\prime}\right) / b^{\prime} \leq \tau^{*} / \zeta-1\right\}$ are the states in which the new generation has to cover a deficit, but participates nonetheless. Further, $D_{2}\left(\tau^{*}\right)$ consists of the states in which $r^{\prime} \geq r^{*}$, so the new generation only has to pay the regular contribution $\zeta$. By definition we have $D_{1}\left(\tau^{*}\right) \cup D_{2}\left(\tau^{*}\right)=D\left(\tau^{*}\right)$, the set of next-period states in which the system continues to exist. Finally, $D^{c}\left(\tau^{*}\right)=\left\{r^{\prime}, b^{\prime} \mid\left(r^{*}-r^{\prime}\right) / b^{\prime}>\tau^{*} / \zeta-1\right\}$, i.e. all the states in which the system collapses.

As Beetsma et al. (2012) show, the belief $\tau^{*}=\zeta$ is a stable equilibrium. As in the PAYG example, the $\Delta^{p}$-function is zero and downward sloping at the minimum contribution, now $\tau=\zeta$, and negative at the maximum contribution $y$. This again implies that besides the trivial minimum contribution, the $\Delta^{p}$-function has an even number of roots in (unstable, stable)-combinations.

Figure 2 shows $\Delta^{p}$ for values of $\tau$ ranging from 0.1 to 0.4 , assuming a basic contribution of

\footnotetext{
${ }^{3}$ Notice that, initially, $a_{0}+\tau_{0}-\theta_{0} / b_{0}=\zeta$, since $a_{0}=\theta_{0}=0$ and $\tau_{0}=\zeta$. Hence, if $r_{1} \leq r^{*}, a_{1}+\tau_{1}-\theta_{1} / b_{1}=$ $\left(1+r_{1}\right) \zeta / b_{1}+\zeta+\left(r^{*}-r_{1}\right) \zeta / b_{1}-\left(1+r^{*}\right) \zeta / b_{1}=\zeta$, while if $r_{1}>r^{*}, a_{1}+\tau_{1}-\theta_{1} / b_{1}=\left(1+r_{1}\right) \zeta / b_{1}+\zeta-\left(1+r_{1}\right) \zeta / b_{1}=\zeta$. This extends to all future $t$.
} 
$\zeta=0.1$ and a minimum return $r^{*}=0.25$ guaranteed by the pension system, as long as it does not collapse. For this parameter constellation, the function $\Delta^{p}(\tau)$ has three roots: the trivial one at $\tau=\zeta=0.1$, an unstable root at 0.18 and a stable root at 0.20 . Given our parameters, the probability that the total contribution exceeds 0.1 is only $6.9 \%$ and the probability that the total contribution exceeds 0.2 is negligible. The reason is that the system only promises a return of $25 \%$ over 30 years, whereas the mean return over this period is $357 \%$. Depending on the selected threshold the system collapses immediately if the contribution is higher than $\zeta$ (with probability of $6.9 \%$ ), i.e. the equilibrium threshold is at the lower stable root, or a collapse is highly unlikely, i.e. the equilibrium threshold is at the higher stable root. These thresholds and probabilities are mostly determined by the level of risk aversion, the rate of time preference and the financial uncertainty. The demographic uncertainty only has a second-order effect. Doubling the variance of the birth rate without changing the mean birth rate has no measurable effect on the thresholds.

These findings are in line with those of Beetsma et al. (2012). However, their only source of uncertainty was the return on savings, so their maximum contribution was $\zeta\left(1+r^{*}\right)$, well below our higher equilibrium threshold. They concluded that the maximum contribution was an equilibrium threshold. Hence, given that the contribution could not exceed this threshold, in their analysis the system would never collapse.

\subsection{Minimum return with buffer}

As a third example we discuss the case in which the pension fund again guarantees a minimum return on the basic contribution $\zeta$, but also maintains a minimum amount of reserves equal to a fraction $\alpha \geq 0$ of the basic contribution. That is, after the cash in- and outflows have taken place in a given period, the fund's assets per young must be equal to $(1+\alpha) \zeta$. The pension benefits are the same as in the previous example, i.e. they are given by (22). Hence, as long as the system persists, the participants again receive a minimum return $r^{*}$ on their contribution. To ensure the fund's asset level of $(1+\alpha) \zeta$, the total contribution must be equal to

$$
\tau_{t}= \begin{cases}\zeta+\alpha \zeta\left[1-\left(1+r_{t}\right) / b_{t}\right] & \text { if } r_{t}>r^{*} \\ \zeta+\left(r^{*}-r_{t}\right) \zeta / b_{t}+\alpha \zeta\left[1-\left(1+r_{t}\right) / b_{t}\right] & \text { if } r_{t} \leq r^{*}\end{cases}
$$

Hence, they differ from the contributions (23) in the previous example by the term $\alpha \zeta[1-(1+$ $\left.\left.r_{t}\right) / b_{t}\right]$, which depends on the reserve requirement $\alpha$. If the new cohort decides to participate, then the payout of the system with and without a buffer is the same, but their contribution differs. However, if the system collapses because the young generation decides not to participate, every member of the old generation receives

$$
\theta_{t}^{c}=\left(1+r_{t}\right)(1+\alpha) \zeta
$$

Hence, the old are better off than under a collapse of the previous system, since now they also receive the buffer.

The introduction of a buffer affects the contribution in various ways. First, the effect of a buffer on the required contribution (standardised by $\zeta$ ) depends on the ratio of the gross return on in- 
vestments and the birth rate:

$$
\frac{\partial\left(\tau_{t} / \zeta\right)}{\partial \alpha}=1-\frac{1+r_{t}}{b_{t}}
$$

For realisations of $1+r_{t}$ above the birth rate $b_{t}$ the presence of buffers actually lowers the required contribution paid by the young. The difference between the required contribution per young in a system with a buffer $(\alpha>0)$ and a system without a buffer consists of two parts, namely in the case of a buffer the young have to inject an additional $\alpha \zeta$ into the system, but they inherit $\left(1+r_{t}\right) \alpha \zeta / b_{t}$. The inheritance is the gross return on the buffer in the previous period divided by the gross population growth. If the size of the young generation is larger relative to the old generation, then the inheritance per young person falls. Whenever the inherited buffer exceeds the required buffer $\alpha \zeta$, the net effect $\alpha \zeta\left[1-\left(1+r_{t}\right) / b_{t}\right]$ is negative and the contribution of the young is lower than in a system without a buffer.

A second effect of a buffer is that it increases the sensitivity of the contribution to the return on investments. The slope of the standardised total contribution depends on the region:

$$
\frac{\partial\left(\tau_{t} / \zeta\right)}{\partial r_{t}}=\left\{\begin{array}{ll}
-\alpha / b_{t}<0 & \text { for } r_{t}>r^{*} \\
-(1+\alpha) / b_{t}<0 & \text { for } r_{t} \leq r^{*}
\end{array} .\right.
$$

In the case of a buffer, a higher return always lowers the total contribution, even if $r_{t}>r^{*}$. This is due to the fact that any additional return on the buffer is not needed to finance the pension payouts, but can be used to lower the total contribution of the young. Higher buffer requirements reinforce this effect,

$$
\frac{\partial^{2}\left(\tau_{t} / \zeta\right)}{\partial r_{t} \partial \alpha}=-\frac{1}{b_{t}}<0
$$

and increase the sensitivity of the total contribution to financial shocks.

Differentiating the contribution with respect to the gross rate of population growth yields:

$$
\frac{\partial\left(\tau_{t} / \zeta\right)}{\partial b_{t}}=\left\{\begin{array}{ll}
\frac{\alpha\left(1+r_{t}\right)}{b_{t}^{2}}>0 & \text { for } r_{t}>r^{*} \\
\frac{(1+\alpha)\left(1+r_{t}\right)-\left(1+r^{*}\right)}{b_{t}^{2}} \gtrless 0 & \text { for } r_{t} \leq r^{*}
\end{array} .\right.
$$

Let $r_{t}>r^{*}$. The smaller is the young generation, i.e. the lower is $b_{t}$, the more buffer there is per young person, hence the lower the total contribution can be. If $r_{t} \leq r^{*}$, there are two cases. One is when $\left(1+r_{t}\right)(1+\alpha) \zeta>\left(1+r^{*}\right) \zeta$, hence there are buffers left in the fund after the old have received their benefit and, hence, a smaller new generation is better off since they can divide these buffers over a smaller group. The other case is when $\left(1+r_{t}\right)(1+\alpha) \zeta<\left(1+r^{*}\right) \zeta$. In this case, the new generation prefers a big cohort (high birth rate), so that the burden of replenishing the fund can be distributed over more individuals.

In this example,

$$
\begin{aligned}
\mathcal{U}^{p}\left(\tau, \tau^{*}\right)= & \max _{s} u(y-s-\tau)+\beta \int_{D_{1}\left(\tau^{*}\right)} u\left[\left(1+r^{\prime}\right) s+\left(1+r^{*}\right) \zeta\right] p\left(\phi^{\prime}\right) \\
& +\beta \int_{D_{2}\left(\tau^{*}\right)} u\left[\left(1+r^{\prime}\right)(s+\zeta)\right] p\left(\phi^{\prime}\right)+\beta \int_{D^{c}\left(\tau^{*}\right)} u\left[\left(1+r^{\prime}\right)(s+(1+\alpha) \zeta)\right] p\left(\phi^{\prime}\right),
\end{aligned}
$$



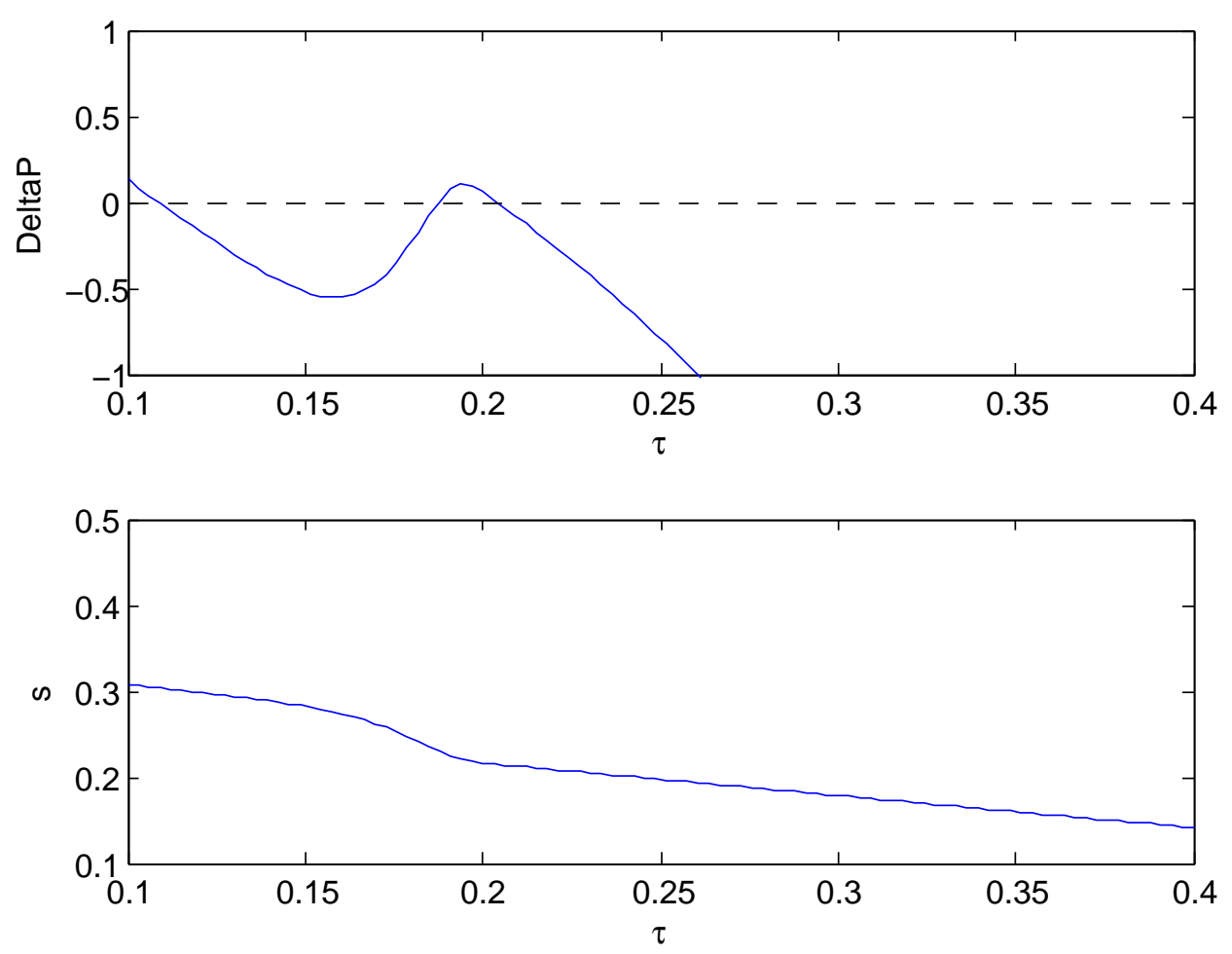

Figure 3: $\Delta(\tau)$ and savings for the funded system with a buffer

where $D_{1}\left(\tau^{*}\right)$ consists of all combinations of $r^{\prime}$ and $b^{\prime}$ such that $r^{\prime} \leq r^{*}$ and $\left(r^{*}-r^{\prime}\right) / b^{\prime}+\alpha\left[1-\left(1+r^{\prime}\right) / b^{\prime}\right] \leq$ $\tau^{*} / \zeta-1$ and $D_{2}\left(\tau^{*}\right)$ consists of all combinations of $r^{\prime}$ and $b^{\prime}$ such that $r^{\prime}>r^{*}$ and $\alpha\left[1-\left(1+r^{\prime}\right) / b^{\prime}\right] \leq$ $\tau^{*} / \zeta-1$. Hence, $D_{1}\left(\tau^{*}\right) \cup D_{2}\left(\tau^{*}\right)$ is the set of all combinations of $r^{\prime}$ and $b^{\prime}$ such that the contribution threshold is not exceeded and the system persists. As before, $D^{c}\left(\tau^{*}\right)$ is the complement of $D_{1}\left(\tau^{*}\right) \cup D_{2}\left(\tau^{*}\right)$. Note that for $\tau^{*}<\zeta(1+\alpha)$ the system may collapse even if $r^{\prime}>r^{*}$. This happens if the new cohort is relatively large, hence the existing buffers are relatively low in per-capita terms, so that the new cohort has to contribute more than $\zeta$ to ensure sufficient reserves. This new generation may not be willing to do so since there are outcomes, namely when $r^{\prime} \leq r^{*}$ and the system does not collapse, in which they only get $\left(1+r^{\prime}\right) \zeta$.

Figure 3 shows $\Delta^{p}$ for values of $\tau$ ranging from 0.1 to 0.4 , assuming a buffer $\alpha=0.1$ and, as before, a basic contribution $\zeta$ of $10 \%$ of the initial endowment and a minimum return $r^{*}$ of $25 \%$. The basic contribution $\zeta=0.10$ does not correspond to an equilibrium threshold since $\Delta^{p}$ is positive. If the system continues, the return on this contribution is at least the return on private savings. If the next generation does not participate, the return on this contribution is higher than the return on private savings since the current generation can then also claim the buffers. For this parameter constellation, the function $\Delta^{p}(\tau)$ has three roots: one at $\tau=0.109$, hence slightly less then $(1+\alpha) \zeta$, an unstable root at 0.19 and a stable root at 0.204 . There is a $5.5 \%$ chance of a higher total contribution than 0.109 , while the chance that the required contribution is higher than the high threshold of 0.204 is again negligible. The lower stable root corresponds to the left-corner solution in the previous example without a buffer. Compared to that situation, the probability of a collapse is lower in this case.

Table 1 shows the numerically computed stable thresholds and probabilities of a collapse of the 


\begin{tabular}{ccccc} 
& 1st stable threshold & Prob. of collapse & 2nd stable threshold ${ }^{*}$ & Prob. of collapse \\
\hline$\alpha$ & & & & \\
$0 \%$ & $10.0 \%$ & $6.9 \%$ & $20.3 \%$ & $0.0 \%$ \\
$10 \%$ & $10.8 \%$ & $5.5 \%$ & $20.3 \%$ & $0.0 \%$ \\
$20 \%$ & $11.7 \%$ & $4.5 \%$ & - & - \\
$30 \%$ & $12.5 \%$ & $3.7 \%$ & - & - \\
\hline$r^{*}$ & & & & - \\
$0 \%$ & $10.8 \%$ & $2.9 \%$ & - & $0.0 \%$ \\
$20 \%$ & $10.8 \%$ & $4.9 \%$ & $19.9 \%$ & $0.0 \%$ \\
$40 \%$ & $10.9 \%$ & $7.4 \%$ & $21.7 \%$ & $0.0 \%$ \\
$70 \%$ & $10.9 \%$ & $12.0 \%$ & $24.3 \%$ & $0.0 \%$ \\
$100 \%$ & $10.9 \%$ & $17.2 \%$ & $26.7 \%$ & - \\
\hline$\rho$ & & & & - \\
1.0 & $10.0 \%$ & $7.4 \%$ & - & $0.0 \%$ \\
3.0 & $10.4 \%$ & $6.3 \%$ & - & $0.0 \%$ \\
5.0 & $10.8 \%$ & $5.5 \%$ & $20.3 \%$ & \\
7.0 & $10.9 \%$ & $5.3 \%$ & $34.2 \%$ & \\
\hline
\end{tabular}

*as percentage of initial endowment $y$

Table 1: Thresholds and probabilities of a collapse of the system for various parameter settings.

above system for various parameter settings. We vary respectively the buffer size $\alpha$, the minimum return $r^{*}$ and the risk aversion $\rho$, each time keeping all the other parameters at their baseline levels given by $\alpha=0.1, \zeta=0.1, \rho=5$ and $r^{*}=0.25$. There are never more than two stable thresholds, while in some cases there is only one. The first block shows that increasing the buffer leads to a lower probability of a collapse, as expected. For relatively low values of $\alpha$, there is a second stable threshold and if the current generation actually has this 'optimistic' belief, the system will (almost) certainly survive. The second block shows the effect of a higher minimum return $r^{*}$. The first (low) threshold is essentially unaffected by higher promised returns. The probability of a collapse increases, because for higher promised returns the next generation is more likely to be confronted with low or even empty buffers that must be replenished.

The last block shows the effect of an increase in the relative risk aversion parameter. As expected, this increases the willingness to participate as indicated by higher thresholds. For already moderately high levels of risk aversion of 3.0 to 5.0, levels generally accepted in the macroeconomic literature, a possibility of 'optimistic' beliefs exists, as there is a high threshold besides the 'pessimistic' low one.

\section{Welfare analysis}

This section analyses for the above three sample arrangements the optimal setting of the policy parameters. For our baseline parameter constellation, all arrangements have two stable equilibrium values for the threshold $\tau^{*}$, a relatively low one and a relatively high one. The question is which of the two equilibrium thresholds is the relevant one. We select the relevant equilibrium threshold by imposing that it be consistent with the participation constraint of the first young generation. This generation is only willing to participate if $\mathcal{U}^{p}\left(\tau_{0}, \tau^{*}\right)>\mathcal{U}^{a}$. This rules out the low threshold. For the PAYG arrangement the low threshold is zero, implying that the first young generation would 
never be willing to pay the old, so that it would never be possible to start a system with mandatory participation. Under the funded scheme without a buffer, the first young would be just willing to pay the contribution rate, but not more than that. Hence, for the low equilibrium threshold, the pension system provides no insurance at all. For the funded scheme with a buffer $\alpha>0$, for any reasonable value of risk aversion, the low equilibrium threshold is slightly below $(1+\alpha) \theta$, the contribution to be made by the first generation. Summarising, if the low equilibrium threshold is the relevant one, it would be impossible to start a pension system that actually provides risk sharing.

Based on these findings, we rule out the low equilibrium threshold and focus on the high equilibrium threshold. ${ }^{4}$ For our PAYG scheme, the high threshold is around $16.6 \%$ of the initial endowment, while the payout is $10 \%$. For our funded systems, the accrual rate is $10 \%$, while the high threshold is roughly $20 \%$. Relative to the expected future benefit these thresholds seem rather high. Moreover, for the high equilibrium threshold the participation constraint is almost never binding and the system would almost never collapse. Obviously, given the stylised nature of the model, we should be careful not to put too much emphasis on the precise values of the thresholds. Introduction of more refinement into the model may well result in more realistic values for the high equilibrium threshold. In particular, the length of retirement is assumed to be the same as the length of the working life. In reality, the former is roughly half of the latter, implying that the same total benefit would correspond to a substantially larger replacement rate of the wage during the active life.

The system designer sets the pension parameters, and these parameters result into a threshold $\tau^{*}$ and a probability of a collapse $P$ in each period. The payout to the current old $\left(\theta_{0}\right)$ and the contribution of the current young $\left(\tau_{0}\right)$ are known at the moment the system is started. If the system collapses, it will remain in autarky forever. We assume that social welfare is the discounted (to period 0 , the moment the system is started) sum of the expected utilities of all generations alive and born in period 0 and later. Of the old generation in period 0 it includes only the utility obtained from their second-period consumption. Given the assumptions just made and using the re-cursiveness of the model, Appendix shows that we can write social welfare $\mathcal{W}$ as:

$$
\mathcal{W}=u\left(\left(1+r_{0}\right) s_{-1}+\theta_{0}\right)+\mathcal{U}^{p}\left(\tau_{0}, \tau^{*}\right)+\beta\left(P V^{a}+(1-P) V^{p}\right)
$$

where

$$
\begin{aligned}
V^{a} & =\frac{b^{a} \mathcal{U}^{a}}{1-\beta b^{e}}, \\
V^{p} & =\frac{1}{1-\beta b^{p}(1-P)}\left(\Gamma^{p}+\beta b^{p} P V^{a}\right),
\end{aligned}
$$

\footnotetext{
${ }^{4}$ In principle one might try to motivate the low equilibrium threshold by events outside the model. For example, the experience of a severe crisis seems to be a good predictor of the emergence of benefit systems. In particular, in the U.S. President Franklin D. Roosevelt presented the Social Security Act of 1935 as a response to the consequences of the Great Depression for vulnerable groups like senior citizens. This response may well have been made acceptable by solidarity feelings towards these groups, even though the initial contributors did not expect to obtain any net benefit from the arrangement. However, we will not follow this route, but require that the participation constraint be fulfilled for all generations, including the first young generation.
} 


\begin{tabular}{ccccccccc}
\hline$\rho$ & \multicolumn{9}{c}{5} & \multicolumn{4}{c}{7} \\
\hline$\mu_{r}$ & \multicolumn{2}{c}{1.27} & \multicolumn{2}{c}{1.22} & \multicolumn{2}{c}{1.27} & 0.22 \\
$\sigma_{r}$ & \multicolumn{2}{c}{0.71} & \multicolumn{2}{c}{0.78} & \multicolumn{2}{c}{0.71} & 0.78 \\
\hline$\mu_{b}$ & 0.317 & 0.312 & 0.317 & 0.312 & 0.317 & 0.312 & 0.317 & 0.312 \\
$\sigma_{b}$ & 0.026 & 0.103 & 0.026 & 0.103 & 0.026 & 0.103 & 0.026 & 0.103 \\
\hline$\theta$ & 0.251 & 0.248 & 0.303 & 0.300 & 0.338 & 0.333 & 0.378 & 0.372 \\
$\tau^{*}$ & 0.298 & 0.296 & 0.413 & 0.411 & 0.514 & 0.512 & 0.641 & 0.640 \\
Compensation & $13.2 \%$ & $13.1 \%$ & $24.5 \%$ & $24.4 \%$ & $39.2 \%$ & $38.9 \%$ & $68.8 \%$ & $68.5 \%$ \\
\hline
\end{tabular}

Table 2: Welfare maximising pension parameters for the PAYG system

where $b^{e}$ is the mean gross population growth rate and $b^{a}\left(b^{p}\right)$ is the gross population growth rate conditional on a (no) collapse in that period. Further, the constant $\Gamma^{p}$ (defined in the Appendix) measures the expected value of the young generation's utility given that the system does not collapse corrected for the gross population growth rate. Hence, we evaluate social welfare for given values of $\theta_{0}$ and $\tau_{0}$. The factor used to discount the utility of future generations is assumed to be the same as the individual discount factor $\beta$.

Now we will turn to the search for the optimal pension arrangements under our three sample systems. We start from our benchmark parameter setting and focus on two values for the relative risk aversion parameter $\rho$, namely 5 and 7. This yields two thresholds for each of the systems we consider. Relative to the baseline parameter setting we also consider variations on this baseline in which we raise the uncertainty parameter $\sigma_{r}$ of the financial process by $10 \%$ and/or the uncertainty parameter $\sigma_{b}$ of the birth process by $300 \%$. To compensate for the effect on the expected return, the parameters $\mu_{r}$ and $\mu_{b}$ are correspondingly reduced.

For the PAYG scheme (see Table 2), we see that an increase in financial market uncertainty raises the optimal pension benefit. This outcome is not surprising, because an increase in financial market uncertainty lowers the level of personal retirement assets when financial markets perform poorly. An increase in the pension benefit protects against low resources in retirement. Along with the increase in the optimal pension benefit, we observe an increase in the equilibrium threshold for the contribution rate. The effects of an increase in the uncertainty of the birth rate on the optimal benefit level and the contribution threshold are only marginal, the reason being that the birth rate does not have any direct effect on utility. It only has an effect through the probability of a collapse. An increase in the degree of risk aversion raises the penalty associated with fluctuations in resources during retirement and, hence, a larger stable source of income becomes desirable. As a result, the optimal pension benefit rises and, along with it, the maximum willingness of the young to contribute. The final row reports by how much each generation's endowment must rise in order to raise the welfare level under autarky to that of the pension system under the old endowment of $y=1$. We see that for relatively low risk aversion and low financial risk, the compensation is only $13 \%$. The welfare gains of the pension system in that specific situation are relatively limited. When risk aversion or financial uncertainty increase, these welfare gains also increase. In order to have the same level of welfare as under the optimal PAYG system, private endowments in autarky must go up by $68 \%$.

Tables 3 and 4 report the optimal arrangements for our funded pension systems. In the system with minimum returns but without buffers (Table 3), we see that the optimal promised return $r^{*}$ is 


\begin{tabular}{ccccccccc}
\hline$\rho$ & \multicolumn{9}{c}{5} & \multicolumn{4}{c}{7} \\
\hline$\mu_{r}$ & \multicolumn{2}{c}{1.27} & \multicolumn{2}{c}{1.22} & \multicolumn{2}{c}{1.27} & 0.22 \\
$\sigma_{r}$ & 0.71 & 0.78 & \multicolumn{2}{c}{0.71} & \multicolumn{2}{c}{0.78} \\
\hline$\mu_{b}$ & 0.317 & 0.312 & 0.317 & 0.312 & 0.317 & 0.312 & 0.317 & 0.312 \\
$\sigma_{b}$ & 0.026 & 0.103 & 0.026 & 0.103 & 0.026 & 0.103 & 0.026 & 0.103 \\
\hline$\theta$ & 0.075 & 0.049 & 0.115 & 0.071 & 0.125 & 0.088 & 0.150 & 0.131 \\
$r^{*}$ & 4.149 & 4.799 & 3.253 & 4.209 & 2.972 & 3.563 & 2.517 & 2.678 \\
$\tau^{*}$ & 0.373 & 0.315 & 0.502 & 0.447 & 0.582 & 0.541 & 0.728 & 0.705 \\
Compensation & $26.5 \%$ & $23.6 \%$ & $40.7 \%$ & $38.8 \%$ & $58.0 \%$ & $56.4 \%$ & $92.0 \%$ & $91.3 \%$ \\
\hline
\end{tabular}

Table 3: Welfare maximising pension parameters for the funded minimum return system

in the same ballpark as the expected return of 3.57 over a 30 year horizon. One striking feature of all optimal arrangements is the high threshold for the contribution, especially when compared to the contribution itself. The reason is that the penalty for a failed system is severe. Once the system collapses, the economy will stay in autarky forever. To avoid this, the optimal system is such that the probability of a collapse is negligible. Higher risk aversion lowers the optimal minimum return $r^{*}$. The consequences of a collapse of the fund become more severe if risk aversion higher. Hence, to reduce the chance that the young would need to make such a large contribution to the scheme that they decide to not participate, the minimum return $r^{*}$ has to be reduced. Welfare under the funded system is potentially much higher than under the PAYG system. The main reason is that the PAYG system is relatively expensive due to the low expected population growth rate $1.06 \%$ per annum) compared to the expected financial return (5.2\%).

Table 4 shows that the welfare maximising buffers are significant, except in the case with both high risk aversion and high uncertainty. The buffer has two roles. It acts as a carrot and as a stick. It acts as a stick in that, if the young generation is not willing to participate, the buffer goes to the current old and the young can not benefit from the returns generated by this buffer. Second, it acts as a carrot as it is a source of free money. The first generation foregoes some of the benefit of the system by having them establish the buffer. Ceteris paribus, this lowers their utility, but increases the welfare of future generations and is, therefore, welfare enhancing. These high buffers also allow for high minimum returns. Participation is attractive due to the existence of the buffers, so the promises by the pension system can be larger with bigger buffers.

The last row shows that the mere welfare gains from introducing these buffers are relatively small. The compensation is only 2 to 4 percentage points larger than in the comparable situation for a pension system without buffers. The first generation is worse off since they have to pay for the initial buffers, while future generations are better off since the pension system effectively shifts resources from this first generation to future generations. These two effects on social welfare largely cancel out.

\section{Conclusion}

This paper has studied voluntary participation in pension arrangements. That is, individuals only participate in the arrangement when their participation constraint is fulfilled, i.e. when participation is more attractive than the alternative of autarky. The policy relevance of the participation 


\begin{tabular}{ccccccccc}
\hline$\rho$ & \multicolumn{9}{c}{5} & \multicolumn{4}{c}{7} \\
\hline$\mu_{r}$ & \multicolumn{2}{c}{1.27} & \multicolumn{2}{c}{1.22} & \multicolumn{2}{c}{1.27} & \multicolumn{2}{c}{1.22} \\
$\sigma_{r}$ & \multicolumn{2}{c}{0.71} & \multicolumn{2}{c}{0.78} & \multicolumn{2}{c}{0.71} & 0.78 \\
\hline$\mu_{b}$ & 0.317 & 0.312 & 0.317 & 0.312 & 0.317 & 0.312 & 0.317 & 0.312 \\
$\sigma_{b}$ & 0.026 & 0.103 & 0.026 & 0.103 & 0.026 & 0.103 & 0.026 & 0.103 \\
\hline$\theta$ & 0.031 & 0.009 & 0.033 & 0.018 & 0.054 & 0.035 & 0.145 & 0.127 \\
$r^{*}$ & 9.53 & 26.76 & 11.81 & 17.81 & 7.671 & 10.064 & 2.531 & 2.592 \\
$\alpha$ & 2.116 & 7.128 & 3.324 & 5.005 & 2.060 & 2.638 & 0.380 & 0.373 \\
$\tau^{*}$ & 0.342 & 0.294 & 0.470 & 0.430 & 0.564 & 0.534 & 0.721 & 0.698 \\
Compensation & $29.2 \%$ & $25.8 \%$ & $44.5 \%$ & $41.9 \%$ & $61.5 \%$ & $59.7 \%$ & $94.2 \%$ & $93.3 \%$ \\
\hline
\end{tabular}

Table 4: Welfare maximising pension parameters buffer system (search algorithm)

issue is rapidly increasing, because in many countries young generations are becoming more and more sceptical about whether they will see any return to the contributions they currently make to their pension plans. Once large groups of participants loose their confidence in a system with mandatory participation, it is bound to collapse, as the political pressure to relax the participation requirement becomes too large. When that happens also the benefits of intergenerational risksharing through the system will be lost. Hence, policy-makers face the task of redesigning pension arrangements in such a way that these benefits will be preserved, while the different groups of participants are happy to stay in the system.

We applied our analysis to a number of important examples, namely a PAYG scheme, a pension fund without buffers and a fund with buffers. Our findings have interesting implications for the buffer policies of pension funds. On the one hand, if the asset returns are positive, the buffer creates some 'free' money that can be used to lower the contributions. However, if the asset returns are negative, the incoming cohort not only has to guarantee the pension benefits of the retired, but it also has to replenish the buffer, implying a contribution that is actually higher than in the case without buffers. Overall, the sensitivity of the contributions to the asset returns increases when the buffer requirements are raised.

We confined ourselves to recursive settings and explored equilibria characterised by thresholds on the contribution that young generations are prepared to make. For standard parameter settings each system featured two such equilibria, of which only the one with the higher threshold was consistent with the initial young benefiting from setting up the system. Finally, we also explored the optimal policy parameter settings that maximised social welfare.

The analysis in this paper may be extended into a number of directions. One would be to allow for a realistic number of overlapping generations. For the sustainability of a funded pension scheme it may matter whether we give each cohort of workers the option to quit (and on what terms) or whether we only allow new cohorts to take a once and for all participation decision. We can also study a richer set pension fund policies and how they affect the risk of a collapse of the fund. In particular, the question is how recovery rules for funds in financial distress could be designed, i.e. which cohort (including the retired) bears which part of the recovery burden, to keep all the cohorts on board and to still make it attractive for newcomers to enter. Moreover, allowing for more overlapping generations would enable us to obtain a more realistic quantification of the benefits from participation. 


\section{References}

[1] Ball, L. and N.G. Mankiw, 2007, Intergenerational risk sharing in the spirit of Arrow, Debreu, and Rawls, with applications to social security design, Journal of Political Economy 115, 4, 523-547.

[2] Beetsma, R.M.W.J. and A.L. Bovenberg, 2009, Pensions and intergenerational risk sharing in general equilibrium, Economica 76, 302, 364-386.

[3] Beetsma, R.M.W.J., W.E. Romp, and S.J. Vos, 2012, Voluntary participation and intergenerational risk sharing in a funded pension system, European Economic Review 56, 1310-1324.

[4] Beetsma, R.M.W.J., W.E. Romp and S.J. Vos, 2013, Intergenerational risk sharing, pensions and endogenous labour supply in general equilibrium, Scandinavian Journal of Economics 115, 1, 141-154.

[5] Bohn, H., 1999, Social security and demographic uncertainty: the risk sharing properties of alternative policies, NBER Working Paper, No. 7030.

[6] Bohn, H., 2003, Intergenerational risk sharing and fiscal policy, Mimeo, University of California at Santa Barbara.

[7] Bonenkamp, J. and E.W.M.T. Westerhout, 2013, Intergenerational risk sharing and endogenous labour supply within funded pension schemes, Economica, forthcoming.

[8] Bovenberg, L., R. Koijen, T. Nijman and C. Teulings, 2007, Saving and investment over the life cycle and the role of collective pension funds, De Economist 155, 4, 347-415.

[9] Campbell, J.Y., Chan, Y.L. and L. Viceira, 2003, A multivariate model of strategic asset allocation, Journal of Financial Economics 67, 1, 41-80.

[10] Cui, J., de Jong, F. and E. Ponds, 2011, Intergenerational risk sharing within funded pension schemes, Journal of Pension Economics and Finance 10, 1-29.

[11] Demange, G., 2009, On sustainable pay-as-you-go contribution rules, Journal of Public Economic Theory 11, 4, 493-527.

[12] Demange, G. and G. Laroque, 2001, Social security with heterogeneous populations subject to demographic shocks, Geneva Papers on Risk and Insurance Theory 26, 5-24.

[13] Enders, W. and H. Lapan, 1982, Social security taxation and intergenerational risk sharing, International Economic Review 23, 647-658.

[14] Flanders, H., 1973, Differentiation under the integral sign, American Mathematical Monthly $80,6,615-627$.

[15] Geanakoplos, J., Magill, M. and M. Quinzii, 2004, Demography and the long-run predictability of the stock market, Brookings Papers on Economic Activity 35, 1, 241-326. 
[16] Gollier, C., 2008, Intergenerational risk-sharing and risk-taking of a pension fund, Journal of Public Economics 92, 5-6, 1463-1485.

[17] Gottardi, P. and F. Kubler, 2006, Social security and risk sharing, CESifo Working Paper, No. 1705.

[18] Hassler, J., and A. Lindbeck, 1997, Intergenerational risk sharing, stability and optimality of alternative pension systems, Institute of International Economic Studies Seminar Paper, No. 631.

[19] Krueger, D. and F. Kubler, 2006, Pareto improving social security reform when financial markets are incomplete!?, American Economic Review 96, 3, 737-755.

[20] Kuhle, W., Ludwig, W. and A. Borsch-Supan, 2007, Demographic change and the equity premium, Mimeo, University of Mannheim.

[21] Matsen, E. and O. Thogerson, 2004, Designing social security - a portfolio choice approach, European Economic Review 48, 883-904.

[22] Mehlkopf, R., 2012, Risk sharing with the unborn, Ph.D thesis, Tilburg University.

[23] Menil, G. de and E. Sheshinski, 2003, The optimal balance of intergenerational transfers and funded pensions in the presence of risk, Mimeo, EHESS and Hebrew University.

[24] Olovsson, C., 2010, Quantifying the risk-sharing welfare gains of social security, Journal of Monetary Economics 57, 364-375.

[25] Penalva, J. and J. van Bommel, 2011, The governance of perpetual financial intermediaries, available at http://dx.doi.org/10.2139/ssrn.1787986.

[26] Prescott, E.C. and J.-V. Rios-Rull, 2005, On equilibrium for overlapping generations organizations, International Economic Review 46, 4, 1065-1080.

[27] Sanchez-Marcos, V. and A.R. Sanchez-Martin, 2006, Can social security be welfare improving when there is demographic uncertainty? Journal of Economic Dynamics and Control 30, 16151646.

[28] Thogersen, 0., 1998, A note on intergenerational risk sharing and the design of pay-as-you-go pension programs, Journal of Population Economics 11, 373-378.

[29] Wagener, A., 2004, On intergenerational risk sharing within social security schemes, European Journal of Political Economy 20, 181-206.

[30] Westerhout, E., 2009, Intergenerational risk sharing in time-consistent pension schemes, Mimeo, CPB Netherlands Bureau for Economic Policy Analysis.

[31] Yu, Y., 2002, Population growth and real asset returns, Ph.D thesis, The Ohio State University. 


\section{A Proof of Equation (14)}

The main difficulty to determine the derivative of $U^{p}\left(\tau, \tau^{*}\right)$ with respect to $\tau^{*}$ lies in the fact that $\tau^{*}$ appears in the area of integration. For the one-dimensional case the solution is trivial. One can use Leibniz's rule for differentiation under the integral sign. In the current higher-dimensional case the solution closely follows Flanders (1973).

Define the function

$$
U^{p}\left(\tau, \tau^{*}\right)=w(\tau)+\int_{\left\{\phi^{\prime} \mid \tau\left(\phi^{\prime}\right) \leq \tau^{*}\right\}} u^{c}\left(\phi^{\prime}\right) p\left(\phi^{\prime}\right)+\int_{\left\{\phi^{\prime} \mid \tau\left(\phi^{\prime}\right)>\tau^{*}\right\}} u^{p}\left(\phi^{\prime}\right) p\left(\phi^{\prime}\right),
$$

where $w$ is the indirect felicity function in the first period of life and $u^{c}$ and $u^{p}$ are the indirect felicity functions under a collapse, respectively no collapse, in the second period of life. The two areas of integration span the entire space $\left\{\phi^{\prime}\right\}$, so we can rewrite $U^{p}\left(\tau, \tau^{*}\right)$ as

$$
U^{p}\left(\tau, \tau^{*}\right)=w(\tau)+\int_{\left\{\phi^{\prime}\right\}} u^{c}\left(\phi^{\prime}\right) p\left(\phi^{\prime}\right)+\int_{\left\{\phi^{\prime} \mid \tau\left(\phi^{\prime}\right)>\tau^{*}\right\}}\left[u^{p}\left(\phi^{\prime}\right)-u^{c}\left(\phi^{\prime}\right)\right] p\left(\phi^{\prime}\right) .
$$

The first two terms do not involve $\tau^{*}$. Only the area of integration in the third term depends on $\tau^{*}$. For the sake of readability define $f\left(\phi^{\prime}\right) \equiv\left[u^{p}\left(\phi^{\prime}\right)-u^{c}\left(\phi^{\prime}\right)\right] p\left(\phi^{\prime}\right)$, the area of integration $C\left(\tau^{*}\right) \equiv$ $\left\{\phi^{\prime} \mid \tau\left(\phi^{\prime}\right)>\tau^{*}\right\}$ and $q\left(\tau^{*}\right) \equiv \int_{C\left(\tau^{*}\right)} f\left(\phi^{\prime}\right)$. That way we have

$$
\frac{\partial U^{p}}{\partial \tau^{*}}=q^{\prime}\left(\tau^{*}\right)=\frac{d}{d \tau^{*}} \int_{C\left(\tau^{*}\right)} f\left(\phi^{\prime}\right) .
$$

Now define a time-varying ${ }^{5}$ mapping from a fixed domain $D$ to $C\left(\tau^{*}\right)$ such that each element $\phi^{\prime} \in$ $C\left(\tau^{*}\right)$ is given by $\phi\left(x, \tau^{*}\right)$, with $x \in D$. Now, we can define the Jacobian matrix of this function

$$
\frac{\partial \phi}{\partial x}=\left[\frac{\partial \phi^{i}}{\partial x^{j}}\right]
$$

The inverse is $\left[\partial x^{j} / \partial \phi^{i}\right]$ and the determinant $|\partial \phi / \partial x|$, which is usually know as the Jacobian. With this we can define velocity $z \equiv \partial \phi / \partial \tau^{*}$. Later we will need the derivative of the Jacobian with respect to $\tau^{*}$

$$
\begin{aligned}
\frac{d}{d \tau *}\left|\frac{\partial \phi}{\partial x}\right| & =\left|\frac{\partial \phi}{\partial x}\right| \operatorname{trace}\left(\left[\frac{\partial \phi}{\partial x}\right]^{-1} \frac{d}{d \tau *}\left[\frac{\partial \phi}{\partial x}\right]\right) \\
& =\left|\frac{\partial \phi}{\partial x}\right| \operatorname{trace}\left(\left[\frac{\partial x^{j}}{\partial \phi^{k}}\right]\left[\frac{\partial z^{i}}{\partial x^{j}}\right]\right) \\
& =\left|\frac{\partial \phi}{\partial x}\right| \sum_{i, j} \frac{\partial x^{j}}{\partial \phi^{i}} \frac{\partial z^{i}}{\partial x^{j}}=\left|\frac{\partial \phi}{\partial x}\right| \sum_{i} \frac{\partial z^{i}}{\partial \phi^{i}} \\
& =\left|\frac{\partial \phi}{\partial x}\right| \operatorname{div}(z),
\end{aligned}
$$

where we have used Jacobi's formula in the first step.

\footnotetext{
${ }^{5}$ The usual interpretation of the variable that shifts the area of integration is time, hence the standard term 'timevarying' mapping.
} 
By the change-of-variables rule we have

$$
\int_{C\left(\tau^{*}\right)} f\left(\phi^{\prime}\right)=\int_{D} f\left(\phi\left(x, \tau^{*}\right)\right)\left|\frac{\partial \phi}{\partial x}\right|
$$

which is a normal fixed domain integral. Normal differentiation and (30) give

$$
\begin{aligned}
\frac{d}{d \tau^{*}} \int_{C\left(\tau^{*}\right)} & f\left(\phi^{\prime}\right)=\int_{D}\left\{\sum \frac{\partial f}{\partial \phi^{i}} z^{i}\left|\frac{\partial \phi}{\partial x}\right|+f\left(\phi^{\prime}\right) \operatorname{div}(z)\left|\frac{\partial \phi}{\partial x}\right|\right\} \\
\ldots & =\int_{C\left(\tau^{*}\right)}\left\{\sum \frac{\partial f}{\partial \phi^{i}} z^{i}+f\left(\phi^{\prime}\right) \operatorname{div}(z)\right\} \\
& =\int_{C\left(\tau^{*}\right)}\left\{\left[\operatorname{grad} f\left(\phi^{\prime}\right)\right] \cdot z+f\left(\phi^{\prime}\right) \operatorname{div}(z)\right\} \\
& =\int_{C\left(\tau^{*}\right)} \operatorname{div}\left(f\left(\phi^{\prime}\right) z\right),
\end{aligned}
$$

with ' $'$ ' the usual dot-product and where going from the first to the second line we have changed back to the time-varying domain. Now, use the divergence theorem to write the last expression as ${ }^{6}$

$$
\int_{\partial C\left(\tau^{*}\right)} f\left(\phi^{\prime}\right) z \cdot d \sigma
$$

with $d \sigma$ the vectorial area element on the surface and $\partial C\left(\tau^{*}\right)$ the boundary of $C\left(\tau^{*}\right)$.

Finally, divide the boundary $\partial C\left(\tau^{*}\right)$ in two parts: $\left\{\phi^{\prime} \mid \tau\left(\phi^{\prime}\right)<\tau^{*}\right\}$ and $\left\{\phi^{\prime} \mid \tau\left(\phi^{\prime}\right)=\tau^{*}\right\}$. In the first part, the integral is zero since $C\left(\tau_{1}^{*}\right) \subseteq C\left(\tau_{2}^{*}\right)$ for $\tau_{1}^{*} \leq \tau_{2}^{*}$, hence any element in this part of the boundary did not move (or moved orthogonal to the surface) so $z \cdot d \sigma=0$. This leaves only the second part of the boundary $\left\{\phi^{\prime} \mid \tau\left(\phi^{\prime}\right)=\tau^{*}\right\}$ and we have

$$
\frac{d}{d \tau^{*}} \int_{C\left(\tau^{*}\right)} f\left(\phi^{\prime}\right)=\int_{\left\{\phi^{\prime} \mid \tau\left(\phi^{\prime}\right)=\tau^{*}\right\}} f\left(\phi^{\prime}\right) z \cdot d \sigma
$$

Switching back to the original notation we have

$$
\frac{\partial U^{p}}{\partial \tau^{*}}=\int_{\left\{\phi^{\prime} \mid \tau\left(\phi^{\prime}\right)=\tau^{*}\right\}}\left[u^{c}\left(\phi^{\prime}\right)-u^{p}\left(\phi^{\prime}\right)\right] p\left(\phi^{\prime}\right) \cdot z,
$$

which is the expression in the text.

\section{B Proof of Slope PAYG}

Start with the definition of $\Delta^{p}$ (see (21))

$$
\Delta^{p}(\tau)=\max _{s} U(y-s-\tau)+\beta\left[1-P_{b}(\theta / \tau)\right] \mathrm{E}_{r}\left(U\left[\left(1+r^{\prime}\right) s+\theta\right]\right)+\beta P_{b}(\theta / \tau) \mathrm{E}_{r}\left(U\left[\left(1+r^{\prime}\right) s\right]\right) .
$$

\footnotetext{
${ }^{6}$ If $C\left(\tau^{*}\right)$ is unbounded, we need a generalised version of the divergence theorem. We ignore this complication. Sufficient is that $f\left(\phi^{\prime}\right)$ vanishes fast enough so that the integral and derivative exist.
} 
The envelop theorem gives

$$
\frac{\partial \Delta^{p}}{\partial \tau}=-U^{\prime}(y-s-\tau)+\beta \frac{\theta}{\tau^{2}} p_{b}(\theta / \tau)\left[\mathrm{E}_{r}\left(U\left[\left(1+r^{\prime}\right) s+\theta\right]\right)-\mathrm{E}_{r}\left(U\left[\left(1+r^{\prime}\right) s\right]\right)\right] .
$$

The slope at the right endpoint is simple. To prevent negative consumption in the second period in the case that the system collapses, savings $s$ must be positive, so any $\tau>y$ is excluded. This implies that the maximum $\tau$ is $y$ and savings are zero. If $\tau \rightarrow y$, the fraction $\theta p_{b}(\theta / \tau) / \tau$ converges to a finite number, while the marginal utility when young goes to infinity, so the slope at the right endpoint goes to minus infinity.

The slope at $\tau=0$ depends on the fraction $\theta p_{b}(\theta / \tau) / \tau^{2}$ as $\tau \downarrow 0$. For the log-normal distribution we have

$$
\begin{aligned}
\frac{\theta}{\tau^{2}} p_{b}(\theta / \tau) & =\frac{\theta}{\tau^{2}} \frac{\tau}{\theta \sqrt{2 \pi \sigma^{2}}} \exp \left[-\frac{1}{2 \sigma^{2}}\left(\log \theta-\log \tau-\mu_{b}\right)^{2}\right] \\
& =\frac{e^{\mu_{b}}}{\theta \sqrt{2 \pi \sigma^{2}}} \frac{\theta}{\tau} e^{-\mu_{b}} \exp \left[-\frac{1}{2 \sigma^{2}}\left(\log \theta-\log \tau-\mu_{b}\right)^{2}\right] \\
& =\frac{e^{\mu_{b}}}{\theta \sqrt{2 \pi \sigma^{2}}} \exp \left[\log \theta-\log \tau-\mu_{b}\right] \exp \left[-\frac{1}{2 \sigma^{2}}\left(\log \theta-\log \tau-\mu_{b}\right)^{2}\right] \\
& =\frac{e^{\mu_{b}}}{\theta \sqrt{2 \pi \sigma^{2}}} \exp \left[\left(\log \theta-\log \tau-\mu_{b}\right)-\frac{1}{2 \sigma^{2}}\left(\log \theta-\log \tau-\mu_{b}\right)^{2}\right] \\
& =\frac{e^{\mu_{b}}}{\theta \sqrt{2 \pi \sigma^{2}}} \exp \left[\left(\log \theta-\log \tau-\mu_{b}\right)\left(1-\frac{1}{2 \sigma^{2}}\left(\log \theta-\log \tau-\mu_{b}\right)\right)\right]
\end{aligned}
$$

For the limit $\tau \downarrow 0$ we have

$$
\lim _{\tau \downarrow 0} \frac{\theta}{\tau^{2}} p_{b}(\theta / \tau)=\frac{e^{\mu_{b}}}{\theta \sqrt{2 \pi \sigma^{2}}} \lim _{\tau \downarrow 0} \exp \left[\left(\log \theta-\log \tau-\mu_{b}\right)\left(1-\frac{1}{2 \sigma^{2}}\left(\log \theta-\log \tau-\mu_{b}\right)\right)\right]=0 .
$$

This implies that the second term in (33) vanishes and we have

$$
\left.\frac{\partial \Delta^{p}}{\partial \tau}\right|_{\tau=0}=-U^{\prime}(y-s)<0
$$

\section{Probability of a collapse and calculation of $U^{p}$ and $\Delta^{p}$}

The main complication in calculating $U^{p}\left(\tau, \tau^{*}\right)$ and $\Delta^{p}(\tau)$ for specific values of $\tau$ is the evaluation of the integrals. In the PAYG model with log-normal random variables (see (21)), this is easy, as we only need to calculate the expected value of second-period utility with respect to the distribution of the interest rate (see Figure 4). This can be done with a Gauss-Hermite integrand.

The other two integrals in the other examples are more difficult since the integrand features a jump. The first step is it to rewrite (12) as

$$
\begin{aligned}
\mathcal{U}^{p}\left(\tau, \tau^{*}\right)=\max _{s} u & (y-s-\tau)+\beta \mathrm{E} u\left[\left(1+r\left(\phi^{\prime}\right)\right) s+\theta\left(\phi^{\prime}\right)\right] \\
& -\beta \int_{D^{c}\left(\tau^{*}\right)}\left\{u\left[\left(1+r\left(\phi^{\prime}\right)\right) s+\theta\left(\phi^{\prime}\right)\right]-u\left[\left(1+r\left(\phi^{\prime}\right)\right) s+\theta^{c}\left(\phi^{\prime}\right)\right]\right\} p\left(\phi^{\prime}\right),
\end{aligned}
$$

where we substituted $c_{1}$ out of the equation and used the fact that $D$ and $D^{c}$ are complements. 
The second term on the right-hand side is simply the expected value of utility when old given that the system always survives, while the third term is, for values of $\phi^{\prime}$ for which the threshold $\tau^{*}$ is exceeded, the difference between the utility with a pay out as if the system continues and the utility with a pay out under a collapse. This equation is useful whenever $D^{c}$ is well-behaved. Alternatively, if $D$ is easier, we can use

$$
\begin{aligned}
\mathcal{U}^{p}\left(\tau, \tau^{*}\right)=\max _{s} u( & y-s-\tau)+\beta \mathrm{E} u\left[\left(1+r\left(\phi^{\prime}\right)\right) s+\theta^{c}\left(\phi^{\prime}\right)\right] \\
& +\beta \int_{D\left(\tau^{*}\right)}\left\{u\left[\left(1+r\left(\phi^{\prime}\right)\right) s+\theta\left(\phi^{\prime}\right)\right]-u\left[\left(1+r\left(\phi^{\prime}\right)\right) s+\theta^{c}\left(\phi^{\prime}\right)\right]\right\} p\left(\phi^{\prime}\right) .
\end{aligned}
$$

In the example of the fund without a buffer, the minimum return, $\theta(\cdot)$ features a kink between $D_{1}$ and $D_{2}$ making low-order Gaussian integrals inaccurate. Rewriting (25) prevents this problem. Using the above rewriting trick gives

$$
\begin{aligned}
\mathcal{U}^{p}\left(\tau, \tau^{*}\right)=\max _{s} u(y-s & -\tau)+\beta \mathrm{E} u\left[\left(1+r^{\prime}\right)(s+\zeta)\right] \\
& +\beta \int_{D_{1}\left(\tau^{*}\right)}\left\{u\left[\left(1+r^{\prime}\right) s+\left(1+r^{*}\right) \zeta\right]-u\left[\left(1+r^{\prime}\right)(s+\zeta)\right]\right\} p\left(\phi^{\prime}\right) .
\end{aligned}
$$

The expected value term is particularly easy to calculate since it does not depend on $b$. The integral itself is more difficult because of the non-square area of integration.

As illustrated in Figure 5, the $D_{1}$ area features a kink where the downward sloping line touches the horizontal axis. To prevent numerical problems we should put $b$ in the inner integral and $r$ in the outer integral. This gives

$$
\begin{aligned}
\mathcal{U}^{p}\left(\tau, \tau^{*}\right) & =\max _{s} u(y-s-\tau)+\beta \mathrm{E} u\left[\left(1+r^{\prime}\right)(s+\zeta)\right] \\
& +\beta \int_{-1}^{r^{*}} \int_{b\left(r^{\prime}, \tau^{*}\right)}^{\infty}\left\{u\left[\left(1+r^{\prime}\right) s+\left(1+r^{*}\right) \zeta\right]-u\left[\left(1+r^{\prime}\right)(s+\zeta)\right]\right\} p\left(b^{\prime}\right) p\left(r^{\prime}\right) d b^{\prime} d r^{\prime}
\end{aligned}
$$

with $b\left(r^{\prime}, \tau^{*}\right)=\left(r^{*}-r^{\prime}\right) /\left(\frac{\tau^{*}}{\zeta}-1\right)$. Now use the fact that the term between curly brackets only

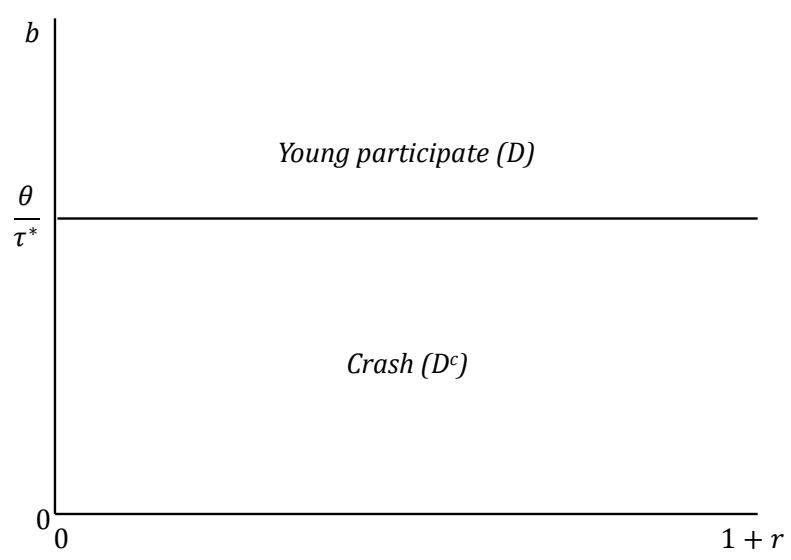

Figure 4: Area of integration for the PAYG example 


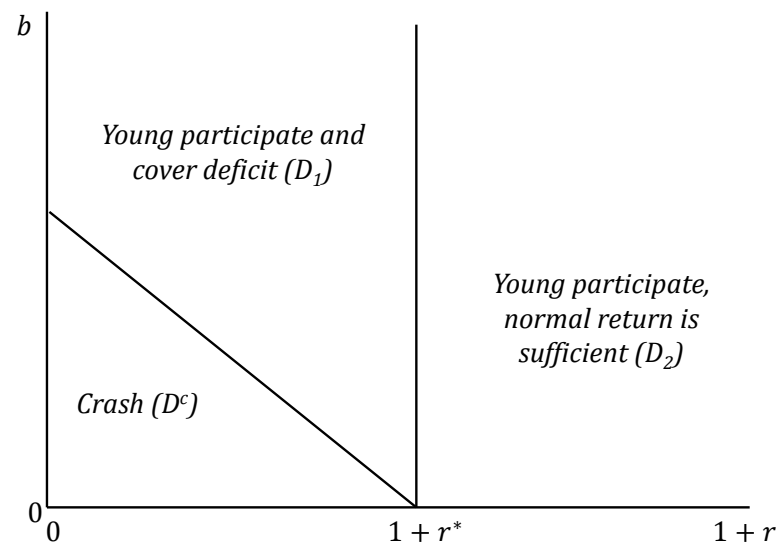

Figure 5: Area of integration for minimum return example

depends on $r^{\prime}$, so we can rewrite this equation as

$$
\begin{aligned}
\mathcal{U}^{p}\left(\tau, \tau^{*}\right) & =\max _{s} u(y-s-\tau)+\beta \mathrm{E} u\left[\left(1+r^{\prime}\right)(s+\zeta)\right] \\
+ & \beta \int_{-1}^{r^{*}}\left[1-P_{b}\left(b\left(r^{\prime}, \tau^{*}\right)\right)\right]\left\{u\left[\left(1+r^{\prime}\right) s+\left(1+r^{*}\right) \zeta\right]-u\left[\left(1+r^{\prime}\right)(s+\zeta)\right]\right\} p\left(r^{\prime}\right) d r^{\prime},
\end{aligned}
$$

where we recall that $P_{b}\left(b\left(r^{\prime}, \tau^{*}\right)\right)$ is the probability that $b^{\prime} \leq b\left(r^{\prime}, \tau^{*}\right)$. The integral can be calculated using any quadrature.

Figure 5 also helps us to determine the sign of the derivative with respect to $\tau^{*}$. The integrand is strictly positive, since in region $D_{1}$ the realised return is smaller than the promised return, $r^{\prime}<r^{*}$. Furthermore, if $\tau^{*}$ increases, the downward sloping line rotates counter-clockwise around point at which it hits the horizontal axis, so $D_{1}$ increases with $\tau^{*}$. Hence, the first derivative of $U^{p}$ with respect to $\tau^{*}$ is positive.

Also for the buffer system, the total area of integration consists of three regions (see Figure 6): the system collapses in $D^{c}$, the minimum return is binding in $D_{1}$ and the market return exceeds the minimum return in $D_{2}$. Areas $D^{c}$ and $D_{1}$ are separated by the line $\left(r^{*}-r\right) / b+\alpha[1-(1+r) / b]=$ $\tau^{*} / \zeta-1$. Simplifying and implicit differentiation gives

$$
\begin{gathered}
\left(1+r^{*}\right)-(1+\alpha)(1+r)=\left(\tau^{*} / \zeta-(1+\alpha)\right) b \Rightarrow \\
\frac{d(1+r)}{d b}=-\frac{\tau^{*} / \zeta-(1+\alpha)}{1+\alpha} .
\end{gathered}
$$

So the slope is determined by the sign of $\tau^{*} / \zeta-(1+\alpha)$. If $\tau^{*}>(1+\alpha) \zeta$, then this line is downward sloping. For low thresholds, i.e. $\tau^{*}<(1+\alpha) \zeta$, the line is upward sloping. In the knife-edge case, i.e. $\tau^{*}=(1+\alpha) \zeta$, the line is vertical. It intersects the $b=0$ boundary in $1+r=\left(1+r^{*}\right) /(1+\alpha)$.

If $\tau^{*}<(1+\alpha) \zeta$, the two areas $D^{c}$ and $D_{2}$ touch at the line $\alpha[1-(1+r) / b]=\tau^{*} / \zeta-1$. Rewriting and implicit differentiation gives the slope 

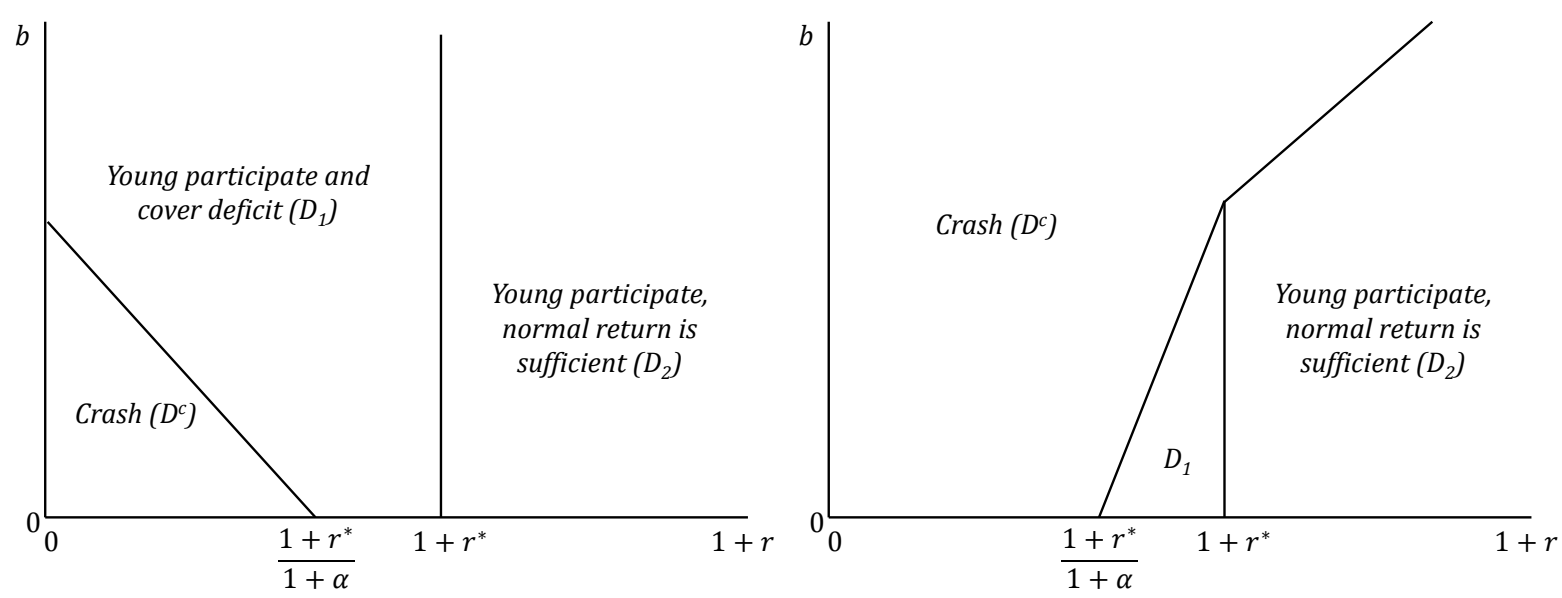

Figure 6: Area of integration for the buffer system with $\tau^{*}>(1+\alpha) \zeta$ (left panel) and $\tau^{*}<(1+\alpha) \zeta$ (right panel)

$$
\begin{aligned}
-\alpha(1+r) & =\left(\tau^{*} / \zeta-(1+\alpha)\right) b \Rightarrow \\
\frac{d(1+r)}{d b} & =-\frac{\tau^{*} / \zeta-(1+\alpha)}{\alpha} .
\end{aligned}
$$

so this line is upward sloping whenever the boundary between $D^{c}$ and $D_{1}$ is upward sloping.

\section{Social welfare}

The system designer sets the pension parameters and these parameters result in a threshold $\tau^{*}$ and a probability of a collapse $P$. The payout to the initial old $\left(\theta_{0}\right)$ and the contribution of the initial young $\left(\tau_{0}\right)$ are known at the time of implementation. If the system collapses, it will stay in autarky forever. These assumptions give for social welfare $\mathcal{W}$

$$
\begin{aligned}
\mathcal{W}=u\left(\left(1+r_{0}\right) s_{-1}\right. & \left.+\theta_{0}\right)+\mathcal{U}^{p}\left(\tau_{0}, \tau^{*}\right) \\
& +\beta\left\{P \mathrm{E}_{0}\left(b_{1} \mid C R_{1}\right) \mathcal{U}^{a}+(1-P) \mathrm{E}_{0}\left(b_{1} \mathcal{U}^{p}\left(\tau_{1}, \tau^{*}\right) \mid N C R_{1}\right)\right\} \\
+ & \beta^{2}\left\{P \mathrm{E}_{0}\left(b_{2} b_{1} \mid C R_{1}\right) \mathcal{U}^{a}+P(1-P) \mathrm{E}_{0}\left(b_{2} b_{1} \mid N C R_{1}, C R_{2}\right) \mathcal{U}^{a}\right. \\
& \left.+(1-P)^{2} \mathrm{E}_{0}\left(b_{2} b_{1} \mathcal{U}^{p}\left(\tau_{2}, \tau^{*}\right) \mid N C R_{1}, N C R_{2}\right)\right\}+\ldots
\end{aligned}
$$

where $C R_{t}$ denotes the event of a collapse in period $t$, while $N C R_{t}$ denotes the event of no collapse in period $t$. For the examples in the text with a funded system (Sections 4.2 and 4.3 ), $\theta_{0}=0$, hence the current old are unaffected by the introduction of a pension system and the first term may be ignored. 
Now, define for all $t \geq 1$ :

$$
\begin{aligned}
b^{a} & =\mathrm{E}_{0}\left(b_{t} \mid C R_{t}\right), \\
b^{p} & =\mathrm{E}_{0}\left(b_{t} \mid N C R_{t}\right), \\
b^{e} & =\mathrm{E}_{0}\left(b_{t}\right), \\
\Gamma^{p} & =\mathrm{E}_{0}\left[b_{t} \mathcal{U}^{p}\left(\tau_{t}, \tau^{*}\right) \mid N C R_{t}\right],
\end{aligned}
$$

where we have dropped the subscripts on the left-hand sides, because of the serial independence of the population growth and because the set up is recursive, hence

$$
\mathrm{E}_{0}\left[\mathcal{U}^{p}\left(\tau_{t}, \tau^{*}\right) \mid N C R_{t}\right]
$$

does not depend on $t \geq 1$. Because $b_{i}$ and $b_{j}$ are independent for different $i$ and $j$ we can use these definitions to write

$$
\begin{aligned}
\mathcal{W}= & u\left(\left(1+r_{0}\right) s_{-1}+\theta_{0}\right)+\mathcal{U}^{p}\left(\tau_{0}, \tau^{*}\right) \\
& +\beta\left\{P b^{a} \mathcal{U}^{a}+(1-P) \Gamma^{p}\right\} \\
& +\beta^{2}\left\{P b^{a} b^{e} \mathcal{U}^{a}+(1-P) P b^{p} b^{a} \mathcal{U}^{a}+(1-P)^{2} b^{p} \Gamma^{p}\right\} \\
& +\beta^{3}\left\{P b^{a} b^{e} b^{e} U^{a}+(1-P) P b^{p} b^{a} b^{e} \mathcal{U}^{a}+P(1-P)^{2} b^{p} b^{p} b^{a} \mathcal{U}^{a}+(1-P)^{3} b^{p} b^{p} \Gamma^{p}\right\} \\
& +\ldots
\end{aligned}
$$

Now, define

$$
\begin{aligned}
V^{a} & =b^{a} \mathcal{U}^{a}\left[1+\beta b^{e}+\beta^{2}\left(b^{e}\right)^{2}+\beta^{3}\left(b^{e}\right)^{3}+\beta^{4}\left(b^{e}\right)^{4}+\beta^{5}\left(b^{e}\right)^{5}+\ldots\right] \\
& =b^{a} \mathcal{U}^{a}+\beta b^{e} V^{a} \\
& =\frac{b^{a} \mathcal{U}^{a}}{1-\beta b^{e}}, \\
V^{p} & =\Gamma^{p}+\beta b^{p}\left[P V^{a}+(1-P) V^{p}\right] \\
& =\frac{1}{1-\beta b^{p}(1-P)}\left[\Gamma^{p}+\beta b^{p} P V^{a}\right],
\end{aligned}
$$

and we can write

$$
\mathcal{W}=u\left(\left(1+r_{0}\right) s_{-1}+\theta_{0}\right)+\mathcal{U}^{p}\left(\tau_{0}, \tau^{*}\right)+\beta\left[P V^{a}+(1-P) V^{p}\right]
$$

which is the equation in the text. 